\title{
Distributions defined by $q$-Supernomials, Fusion Products, and Demazure Modules
}

\author{
Stavros Kousidis \\ Rosenthalstr. 17 \\ 53859 Niederkassel \\ Germany \\ st . kousidis@googlemail.com
}

\author{
Ernst Schulte-Geers \\ Deutschherrenstr. 49 \\ 53177 Bonn \\ Germany \\ ernst.schulteg@t-online.de
}

Submitted: Dec 17, 2014; Accepted: Feb 14, 2015; Published: Mar 6, 2015

Mathematics Subject Classifications: 05A16, 60B99, 06B15

\begin{abstract}
We prove asymptotic normality of the distributions defined by $q$-supernomials, which implies asymptotic normality of the distributions given by the central string functions and the basic specialization of fusion modules of the current algebra of $\mathfrak{s l}_{2}$. The limit is taken over linearly scaled fusion powers of a fixed collection of irreducible representations. This includes as special instances all Demazure modules of the affine Kac-Moody algebra associated to $\mathfrak{s l}_{2}$. Along with an available complementary result on the asymptotic normality of the basic specialization of graded tensors of the type $A$ standard representation, our result is a central limit theorem for a serious class of graded tensors. It therefore serves as an indication towards universal behavior: The central string functions and the basic specialization of fusion and, in particular, Demazure modules behave asymptotically normal, as the number of fusions scale linearly in an asymptotic parameter, $N$ say.
\end{abstract}

Keywords: $q$-supernomial; current algebra; affine Kac-Moody algebra; fusion product; Demazure module; basic specialization; asymptotic normality; central limit theorem; occupancy statistic; mixing distribution

\section{Introduction}

The $q$-supernomial coefficients encode certain integer partitions as polynomials in a variable $q$ of the form

$$
\sum_{j_{1}+\cdots+j_{m}=a} q^{\sum_{i=1}^{m} j_{i}\left(j_{i}+\sum_{\ell=1}^{i-1} L_{\ell}\right)} \prod_{\ell=1}^{m}\left[\begin{array}{c}
L_{\ell}+j_{\ell+1} \\
j_{\ell}
\end{array}\right]_{q}
$$


The $L_{1}, \ldots, L_{m}$ and $a$ are nonnegative integers and $\left[\begin{array}{l}a \\ b\end{array}\right]_{q}$ denotes the well known $q$-binomial coefficient that enumerates inversions in words. They were introduced by Schilling and Warnaar, who studied their symmetries, recurrences and $q$-series limits and gave a combinatorial interpretation as the enumeration of so-called $\left(L_{1}, \ldots, L_{m}\right)$-admissible generalized Durfee dissection partitions with exactly a parts [26].

A natural motivation for the investigation of asymptotic statistical properties of the discrete distributions defined by the coefficients of those polynomials is to understand the qualitative behavior of this special kind of integer partitions for large values of the parameters $L_{1}, \ldots, L_{m} \in \mathbf{Z}_{+}$.

However, our initial motivation for the study of $q$-supernomials is their appearance as Hilbert series of fusion modules of the current algebra $\mathfrak{s l}_{r} \otimes \mathbf{C}[t]$ that were introduced by Feigin and Loktev [11], that is tensor products of irreducible representations endowed with a grading that is encoded by the variable $q$. The coefficients of those Hilbert series (also called string functions) encode dimensions of certain isotropic components called weight spaces. While the exact determination of those coefficients by coefficient extraction is certainly possible in any fixed instance of a $q$-supernomial, the explicit description of those coefficients remains intractable and one usually is satisfied with concrete expressions for their generating function, the $q$-supernomial. For large values of the parameters $L_{1}, \ldots, L_{m}$ one may expect that the asymptotic behavior of the distributions defined by the $q$-supernomials is governed by probabilistic limit theorems, so that precise assertions about "typical" behavior are possible. In this work we show that limit theorems towards asymptotically normal behavior do indeed hold, and deduce the following result for the (central) string functions of fusion modules of the current algebra $\mathfrak{s l}_{2} \otimes \mathbf{C}[t]$ and their so-called basic specialization (a sum of string functions).

Consider a sequence of fusion modules $\left(\mathbf{C}^{2}\right)^{* L_{1}^{N}} * \cdots *\left(\mathbf{C}^{m+1}\right)^{* L_{m}^{N}}$ of the current algebra $\mathfrak{s l}_{2} \otimes \mathbf{C}[t]$. Assume that the exponents grow on a linear scale, i.e. $\left(L_{1}^{N}, \ldots, L_{m}^{N}\right) / N \rightarrow \mathbf{a} \neq 0$ as $N \rightarrow \infty$. Then, the central string functions and basic specialization of those modules behave asymptotically normal with mean and variance growing quadratically and cubically in $N$, respectively, with explicitly calculable leading terms.

We give the precise statements describing the leading terms of this asymptotic expansion of the Hilbert series in Theorem 3.1 and Theorem 3.2. Concerning the asymptotic growth of dominating weight spaces in fusion modules we conjecture that corresponding local limit theorems hold, and that similar results can be shown for the fusion of type $A$ symmetric power representations. Furthermore, we specialize our findings to the case of Demazure modules that have been studied through different methods earlier in the literature.

The material is broadly divided into two parts: the first part is devoted to the probabilistic-combinatorial problem of deriving limit theorems for the q-supernomial distributions, and the second part explains the representation theoretic interpretation of the limit theorems derived in the first part. 


\section{Distributions defined by $q$-supernomials}

Let $\mathbf{L}:=\left(L_{1}, \ldots, L_{m}\right) \in \mathbf{Z}_{+}^{m}, a \in \mathbf{Z}_{+}, j_{m+1}=0$. Consider the $q$-supernomial

$$
\tilde{T}(\mathbf{L}, a)(q)=\sum_{j_{1}+\cdots+j_{m}=a} q^{\sum_{i=1}^{m} j_{i}\left(j_{i}+\sum_{\ell=1}^{i-1} L_{\ell}\right)} \prod_{\ell=1}^{m}\left[\begin{array}{c}
L_{\ell}+j_{\ell+1} \\
j_{\ell}
\end{array}\right]_{q},
$$

that enumerates $a$-restricted $\mathbf{L}$-admissible partitions $[26]^{1}$, and the cumulative generating function of the unrestricted number of $\mathbf{L}$-admissible partitions

$$
\tilde{T}(\mathbf{L})(q):=\sum_{a=0}^{L_{1}+\cdots+L_{m}} \tilde{T}(\mathbf{L}, a)(q) .
$$

We show that the unrestricted number and in certain (typical) cases the $a$-restricted number of $\mathbf{L}$-admissible partitions are asymptotically normally distributed with asymptotic parameter being a convergent sequence $\frac{1}{N} \mathbf{L}^{N}$, as $N \rightarrow \infty$.

\section{$2.1 \quad$ Statistical notions}

Standard sources are $[1,2,12,13]$. All our random variables $X$ will be discrete and finite. Recall that the expected value of such a random variable is the weighted average $\mathbf{E}(X)=$ $\sum_{x} \mathbf{P}(X=x) x$. The covariance of two random variables $X$ and $Y$ is $\operatorname{Cov}(X, Y)=\mathbf{E}((X-$ $\mathbf{E}(X))(Y-\mathbf{E}(Y)))$. They are said to be uncorrelated if $\operatorname{Cov}(X, Y)=0$. The variance of $X$ is $\operatorname{Var}(X)=\operatorname{Cov}(X, X)$. Its probability generating function is $\mathbf{E}\left(q^{X}\right)=\sum_{x} \mathbf{P}(X=x) q^{x}$, and the associated probability distribution $\mu_{X}=\sum_{x} \mathbf{P}(X=x) \delta_{x}$. Here, $\delta_{x}$ denotes the Dirac distribution (point mass) at $x$. A sequence $X_{N}$ converges $\mathbf{P}$-almost surely $(\mathbf{P}-$ a.s. for short) to $X$ if $\mathbf{P}\left(\lim _{N \rightarrow \infty} X_{N}=X\right)=1$. Convergence and equality in distribution will be denoted by $\stackrel{d}{\longrightarrow}$ and $\stackrel{d}{=}$, respectively. $\mathcal{N}(\mu, \Sigma)$ will denote the normal distribution with mean $\mu$ and covariance matrix $\Sigma$. Note that $\mathcal{N}(\mu, 0)=\delta_{\mu}$. The conditional probability $\mathbf{P}(Y=y \mid X=x)=\mathbf{P}(X=x)^{-1} \mathbf{P}(X=x, Y=y)$ is the probability of $Y$ taking the value $y$ given the occurence of the value $x$ for $X$. A mixture of distributions $\mu_{X_{i}}$ is a convex combination thereof, i.e. $\sum_{i} w_{i} \mu_{X_{i}}$ for some weights $w_{i} \geqslant 0$ with $\sum_{i} w_{i}=1$. The probability generating function of a mixture is $\sum_{i} w_{i} \mathbf{E}\left(q^{X_{i}}\right)$.

\subsection{Preliminaries}

The distributions with probability generating function

$$
F_{a, b}(q):=\left[\begin{array}{c}
a+b \\
a
\end{array}\right]_{q} /\left(\begin{array}{c}
a+b \\
a
\end{array}\right)
$$

were first investigated by Mann and Whitney [24], who showed:

\footnotetext{
${ }^{1}$ The notation $\tilde{T}(\mathbf{L}, a)(q)$ is taken from there for consistency.
} 
Theorem 2.1. Let $\operatorname{Inv}_{a, b}$ be a random variable with distribution $F_{a, b}$. Then $\operatorname{Inv}_{a, b}$ has expectation $\mathbf{E}\left(\operatorname{Inv}_{a, b}\right)=\frac{1}{2} a b$, variance $\operatorname{Var}\left(\operatorname{Inv}_{a, b}\right)=\frac{1}{12} a b(a+b+1)$, and as $a, b \rightarrow \infty$ one has

$$
\frac{\operatorname{Inv}_{a, b}-\mathbf{E}\left(\operatorname{Inv}_{a, b}\right)}{\sqrt{\operatorname{Var}\left(\operatorname{Inv}_{a, b}\right)}} \stackrel{d}{\longrightarrow} \mathcal{N}(0,1)
$$

A corresponding local limit theorem was proved by Takacs [30].

Remark 2.2. It is well known that the $q$-binomial $\left[\begin{array}{c}a+b \\ a\end{array}\right]_{q}$ is the generating function for inversions in words of $a$ zeroes and $b$ ones. Consider a word (unordered sequence) $w=\left(w_{1}, \ldots, w_{n}\right)$ of elements from an ordered set. A 4-tuple $\left(i, j, w_{i}, w_{j}\right)$ with $i<j$ and $w_{i}>w_{j}$ is then called an inversion.

Let us also recall the following classical result about the asymptotic normality of multinomial distributions (see e.g. [6]).

Theorem 2.3. Let the sequence $\mathbf{B}^{N}$ have the multinomial distribution with parameters $N$ and $\mathbf{p}=\left(p_{0}, p_{1}, \ldots, p_{m}\right)$. Then, we have mean $\mathbf{E}\left(\mathbf{B}^{N}\right)=N \mathbf{p}$, covariance matrix $\operatorname{Cov}\left(\mathbf{B}^{N}\right)=N \Sigma$, and

$$
\frac{\mathbf{B}^{N}-N \mathbf{p}}{N^{1 / 2}} \stackrel{d}{\longrightarrow} \mathcal{N}(\mathbf{0}, \Sigma),
$$

where $\Sigma=\operatorname{diag}(\mathbf{p})-\mathbf{p}^{t} \mathbf{p}$ (not of full rank).

\subsubsection{Elementary definitions}

We call a vector $\mathbf{j}=\left(j_{1}, \ldots, j_{m}\right) \in \mathbf{Z}_{+}^{m}$ compatible to $\mathbf{L} \in \mathbf{Z}_{+}^{m}$ if $j_{i} \leqslant L_{i}+j_{i+1}$ for $i=1, \ldots m$, and a probability distribution $\mathbf{L}$-compatible if the set of $\mathbf{L}$-compatible values has probability one. For $\mathbf{L}$-compatible $\mathbf{j}$ we let $\operatorname{Inv}(\mathbf{L}, \mathbf{j})$ denote a random variable with probability generating function

$$
F(\mathbf{L}, \mathbf{j})(q):=\prod_{i=1}^{m} F_{L_{i}+j_{i+1}, j_{i}}(q),
$$

where here and in the sequel $j_{m+1}=0$. We let

$$
Q(\mathbf{L}, \mathbf{j})=\sum_{i=1}^{m} j_{i}\left(j_{i}+\sum_{\ell=1}^{i-1} L_{\ell}\right) .
$$

We view the normalized generating function $\tilde{T}(\mathbf{L})(q) / \tilde{T}(\mathbf{L})(1)($ and $\tilde{T}(\mathbf{L}, a)(q) / \tilde{T}(\mathbf{L}, a)(1))$ as a mixture of probability generating functions

$$
\tilde{T}(\mathbf{L})(q) / \tilde{T}(\mathbf{L})(1)=\sum_{\mathbf{j}} q^{Q(\mathbf{L}, \mathbf{j})} F(\mathbf{L}, \mathbf{j})(q) \mathbf{P}(\mathbf{J}=\mathbf{j})
$$


weighted by $\mathbf{P}(\mathbf{J}=\mathbf{j})=\mathbf{P}\left(J_{1}=j_{1}, \ldots, J_{m}=j_{m}\right)=\prod_{i=1}^{m}\left(\begin{array}{c}L_{i}+j_{i+1} \\ j_{i}\end{array}\right) / \tilde{T}(\mathbf{L})(1)$. The implicit dependency on the admission vector $\mathbf{L}$ will be from now on suppressed in the notation for convenience. We furthermore define (the conditional distribution of) a random variable $Y$ by $\mathbf{P}(Y=i \mid \mathbf{J}=\mathbf{j})=\mathbf{P}(\operatorname{Inv}(\mathbf{L}, \mathbf{j})=i)$, so that we can rephrase

$$
\tilde{T}(\mathbf{L})(q) / \tilde{T}(\mathbf{L})(1)=\mathbf{E}\left(q^{Q(\mathbf{L}, \mathbf{J})+Y}\right) .
$$

Consequently, our interest lies in the distribution of the random variable

$$
T=Q(\mathbf{L}, \mathbf{J})+Y
$$

whose asymptotics we will treat by splitting $T$ into an "occupancy part" $Q(\mathbf{L}, \mathbf{J})+\mathbf{E}(Y \mid \mathbf{J})$, dependent only on $\mathbf{J}$, and a remaining "rest-inversion part" $R=Y-\mathbf{E}(Y \mid \mathbf{J})$ "orthogonal" to $\mathbf{J}$, and investigating the two parts individually. We note that by Theorem 2.1 we have

$$
\mathbf{E}(Y \mid \mathbf{J})=\frac{1}{2}\left(\left(L_{m}-J_{m}\right) J_{m}+\sum_{i=1}^{m-1}\left(L_{i}+J_{i+1}-J_{i}\right) J_{i}\right)=: e(\mathbf{L}, \mathbf{J})
$$

so that $\mathbf{E}(Y \mid \mathbf{J})$ is a quadratic function in $\mathbf{J}$. Let us call the distribution of $\mathbf{J}$ the mixing distribution (for the total number of $\mathbf{L}$-admissible partitions). To explain the behavior of mixing distributions and to introduce a convenient way to refer to them, let us describe a simple random experiment. We focus our attention on the unrestricted case first.

\subsubsection{The probabilistic setup for the unrestricted case}

Consider $m$ many mutually independent random sources $\mathcal{S}_{1}, \mathcal{S}_{2}, \ldots, \mathcal{S}_{m}$ emitting words $W(1), \ldots, W(m)$. Each word $W(i)=\left(X_{1}(i), X_{2}(i), \ldots, X_{L_{i}}(i)\right)$ is a sequence of $L_{i}$ many mutually independent letters $X_{k}(i)$, where each $X_{k}(i)$ is uniformly distributed from the alphabet $\{0, \ldots, i\}$. For $0 \leqslant i \leqslant k$ let $B_{i}(k):=\sum_{j=1}^{L_{k}} 1_{\{i\}}\left(X_{j}(k)\right)$ denote the random variable the number of appearances of letter $i$ in word $W(k)$. Then, the random vector $\mathbf{B}(k):=\left(B_{0}(k), \ldots, B_{k}(k)\right)$ is the occupancy (statistic) of word $W(k), S(k):=$ $\sum_{i=1}^{L_{i}} X_{i}(k)=\sum_{j=0}^{k} j B_{j}(k)$ is the sum of word $W(k), \mathbf{B}_{\mathbf{L}}:=(\mathbf{B}(1), \ldots, \mathbf{B}(m))$ is the total occupancy of $W(1), \ldots, W(m)$, and $S_{\mathbf{L}}:=\sum_{i=1}^{m} S(i)$ is called the total sum of words $W(1), \ldots, W(m)$. Clearly, under the assumptions above, $S_{\mathbf{L}}$ is the sum of independent uniformly distributed random variables and we have

$$
\begin{aligned}
\mathbf{E}\left(S_{\mathbf{L}}\right) & =\frac{1}{2} \sum_{i=1}^{m} i L_{i}, \\
\operatorname{Var}\left(S_{\mathbf{L}}\right) & =\frac{1}{12} \sum_{i=1}^{m}(i+2) i L_{i} .
\end{aligned}
$$

The probability distribution of $\mathbf{J}$ arises in this experiment as follows. 
Proposition 2.4. Let $\mathbf{B}_{\mathbf{L}}=(\mathbf{B}(1), \ldots, \mathbf{B}(m))$ be as above. That is, the random vectors $\mathbf{B}(i)=\left(B_{0}(i), \ldots, B_{i}(i)\right)$ are independent, where each $\mathbf{B}(i)$ has a (uniform) multinomial distribution with parameters $L_{i}$ and $p_{0}=\ldots=p_{i}=\frac{1}{i+1}$. Define

$$
J_{i}:=\sum_{k=i}^{m} A_{k-i+1}(k), \text { where } A_{k}(i):=\sum_{j=k}^{i} B_{j}(i) .
$$

Then, the joint distribution of $\left(J_{1}, \ldots, J_{m}\right)$ is

$$
\mathbf{P}\left(J_{1}=j_{1}, \ldots, J_{m}=j_{m}\right)=\prod_{i=1}^{m}\left(\begin{array}{c}
L_{i}+j_{i+1} \\
j_{i}
\end{array}\right) /(i+1)^{L_{i}} .
$$

where $\mathbf{j}=\left(j_{1}, \ldots, j_{m}\right) \in \mathbf{N}_{0}^{m}$ (and with the usual convention about binomial coefficients that $\left(\begin{array}{l}a \\ b\end{array}\right)=0$ unless $\left.0 \leqslant b \leqslant a\right)$. These numbers define a $\mathbf{L}$-compatible probability distribution.

Proof. By formal generating functions it is clear that the joint generating function of $\left(J_{1}, \ldots, J_{m}\right)$ as defined above is

$$
\mathbf{E}\left(t_{1}^{J_{1}} \ldots t_{m}^{J_{m}}\right)=\prod_{i=1}^{m}\left(\sum_{j=0}^{i} \prod_{k=i-j+1}^{i} t_{k}\right)^{L_{i}} /(i+1)^{L_{i}} .
$$

Now extract coefficients to see that this corresponds to the distribution defined above. The $\mathbf{L}$-compatibility is obvious.

Remark 2.5. The proof shows that $\tilde{T}(\mathbf{L})(1)=\prod_{i=1}^{m}(i+1)^{L_{i}}$, which can be seen directly from the combinatorial definition of $q$-supernomials. Furthermore, since $A_{k-i+1}(k)=$ $B_{k-i+1}(k)+B_{k-i+2}(k)+\cdots+B_{k}(k)$ counts the number of appearances of the highest $i$ letters in word $W(k)$, the $J_{i}$ may be described as the overall count of the $i$ highest non-zero letters in all words.

Proposition 2.4 shows that the mixing distribution for the total number of $\mathbf{L}$-admissible partitions may be realized as a simple linear transformation of $\mathbf{B}_{\mathbf{L}}$. We call $\mathbf{B}_{\mathbf{L}}$ the underlying occupancy distribution. This representation can be used for explicit calculations, and reduces the asymptotic treatment of $\mathbf{J}$ in the unrestricted case to the well known asymptotics of multinomial distributions as described in Theorem 2.3.

\subsubsection{The probabilistic setup for the $a$-restricted case}

The same experiment as in $\S 2.2 .2$ describes the $a$-restricted case when we consider only the outcomes with total sum $S_{\mathbf{L}}=a$. That is, for the $a$-restricted case the underlying occupancy distribution is $\mathbf{B}_{\mathbf{L}} \mid S_{\mathbf{L}}=a$, i.e. the distribution of $\mathbf{B}_{\mathbf{L}}$ conditioned by $S_{\mathbf{L}}=a$. In order to have a succinct wording for the "most important" restricted cases we make the following definition (Cf. (10)). 
Definition 2.6. We call the $a$-restricted cases with $a=\mathbf{E}\left(S_{\mathbf{L}}\right)\left(\operatorname{resp} . a=\mathbf{E}\left(S_{\mathbf{L}}\right) \pm \frac{1}{2}\right)$ central.

This terminology is justified due to the symmetry of the distribution of $S_{\mathbf{L}}$ around $\mathbf{E}\left(S_{\mathbf{L}}\right)$, and their central importance due to the strong law of large numbers,

$$
\frac{1}{N} \mathbf{E}\left(S_{\mathbf{L}^{N}}\right) \stackrel{\text { P-a.s. }}{\longrightarrow} \frac{1}{2} \sum_{k=1}^{m} k a_{k}, \text { as } \frac{1}{N} \mathbf{L}^{N} \rightarrow\left(a_{1}, \ldots, a_{m}\right) .
$$

\subsection{General asymptotic considerations}

Throughout this section let $\mathbf{L}^{N}$ be a sequence of admission vectors, and $\mathbf{J}^{N}$ a sequence of $\mathbf{L}^{N}$-compatible mixing distributions. We consider the sequence of inversion statistics

$$
Y^{N} \text {, defined by } \mathbf{P}\left(Y^{N}=i \mid \mathbf{J}^{N}=\mathbf{j}\right)=\mathbf{P}\left(\operatorname{Inv}\left(\mathbf{L}^{N}, \mathbf{j}\right)=i\right) \text {, }
$$

and recall the associated definitions from $§ 2.2 .1$,

$$
\begin{aligned}
T^{N} & =Q\left(\mathbf{L}^{N}, \mathbf{J}^{N}\right)+Y^{N}, \\
R^{N} & =Y^{N}-\mathbf{E}\left(Y^{N} \mid \mathbf{J}^{N}\right), \\
e\left(\mathbf{L}^{N}, \mathbf{J}^{N}\right) & =\mathbf{E}\left(Y^{N} \mid \mathbf{J}^{N}\right) .
\end{aligned}
$$

Let us note the asymptotic behavior of the conditional distribution of $R^{N}$.

Lemma 2.7. If $\frac{1}{N} \mathbf{L}^{N} \rightarrow \mathbf{a} \neq \mathbf{0}$, and $\mathbf{j}^{N}$ is a sequence of $\mathbf{L}^{N}$-compatible vectors such that $\frac{1}{N} \mathbf{j}^{N} \rightarrow \mathbf{b} \neq \mathbf{0}$, then

$$
\frac{\operatorname{Inv}\left(\mathbf{L}^{N}, \mathbf{j}^{N}\right)-\mathbf{E}\left(\operatorname{Inv}\left(\mathbf{L}^{N}, \mathbf{j}^{N}\right)\right.}{N^{3 / 2}} \stackrel{d}{\longrightarrow} \mathcal{N}(0, v(\mathbf{a}, \mathbf{b}))
$$

where $v(\mathbf{a}, \mathbf{b})=\frac{1}{12} \sum_{i=1}^{m}\left(a_{i}+b_{i+1}-b_{i}\right) b_{i}\left(a_{i}+b_{i+1}\right)\left(\right.$ where $\left.b_{m+1}=0\right)$.

Proof. By definition, the random variable

$$
\operatorname{Inv}\left(\mathbf{L}^{N}, \mathbf{j}^{N}\right)-\mathbf{E}\left(\operatorname{Inv}\left(\mathbf{L}^{N}, \mathbf{j}^{N}\right)\right)
$$

is distributed like the sum $\sum_{i=1}^{m} X_{i}^{N}$ of $m$ independent random variables

$$
\left.X_{i}^{N} \stackrel{d}{=} \operatorname{Inv}\left(L_{i}^{N}+j_{i+1}^{N}-j_{i}^{N}, j_{i}^{N}\right)-\frac{1}{2}\left(L_{i}^{N}+j_{i+1}^{N}-j_{i}^{N}\right) j_{i}^{N}\right) .
$$

Let $a_{i}^{N}=L_{i}^{N}+j_{i+1}^{N}-j_{i}^{N}$ and $b_{i}^{N}=j_{i}^{N}$. By assumption the limits $a_{i}=\lim \frac{1}{N} a_{i}^{N}$ and $b_{i}=\lim \frac{1}{N} b_{i}^{N}$ exist. If $a_{i}=0$ or $b_{i}=0$, then $\frac{1}{N^{3 / 2}} X_{i}^{N} \stackrel{d}{\longrightarrow} 0 \stackrel{d}{=} \mathcal{N}(0,0)$. If $a_{i}>0$ and $b_{i}>0$ we find that $\frac{1}{N^{3}} \operatorname{Var}\left(\operatorname{Inv}\left(a_{i}^{N}, b_{i}^{N}\right) \rightarrow \frac{1}{12} a_{i} b_{i}\left(a_{i}+b_{i}\right):=w\left(a_{i}, b_{i}\right)\right.$, and Theorem 2.1 gives

$$
\frac{X_{i}^{N}}{N^{3 / 2}} \stackrel{d}{\longrightarrow} \mathcal{N}\left(0, w\left(a_{i}, b_{i}\right)\right) .
$$

Thus under the conditions above $\frac{1}{N^{3 / 2}} \sum_{i=1}^{m} X_{i}^{N} \stackrel{d}{\longrightarrow} \mathcal{N}\left(0, \sum_{i=1}^{m} w\left(a_{i}, b_{i}\right)\right)=\mathcal{N}(0, v(\mathbf{a}, \mathbf{b}))$. 
Remark 2.8. The case $a_{i} b_{i}=0$ for all $i$ (that is $v(\mathbf{a}, \mathbf{b})=0$ ) is less interesting but not excluded. In this case we interpret $\mathcal{N}(0,0):=\delta_{0}$ as the Dirac-measure (point mass) at 0 .

Next we observe that under mild conditions the limiting distributions of the (normalized) random variables $R^{N}$ and $\mathbf{J}^{N}$ are asymptotically independent.

Theorem 2.9. Let $R(\mathbf{a}, \mathbf{b})$ denote a random variable with distribution $\mathcal{N}(0, v(\mathbf{a}, \mathbf{b}))$. If $\frac{1}{N} \mathbf{L}^{N} \rightarrow \mathbf{a} \neq \mathbf{0}$, and if there exists $\mathbf{b} \in \mathbf{R}_{+}^{m}$ and a positive semidefinite matrix $\Sigma \in \mathbf{R}^{m \times m}$ of positive rank such that

$$
\frac{\mathbf{J}^{N}-N \mathbf{b}}{N^{1 / 2}} \stackrel{d}{\longrightarrow} \mathcal{N}(\mathbf{0}, \Sigma)
$$

Then, as $N \longrightarrow \infty$,

$$
\left(\frac{R^{N}}{N^{3 / 2}}, \frac{\mathbf{J}^{N}-N \mathbf{b}}{N^{1 / 2}}\right) \stackrel{d}{\longrightarrow}(R(\mathbf{a}, \mathbf{b}), \mathcal{N}(\mathbf{0}, \Sigma)),
$$

where the constituents on the right-hand side are independent.

Proof. Let $A \subset \mathbf{R}$ be a Borel set and $f: \mathbf{R}^{m} \rightarrow \mathbf{R}$ be bounded and continuous. We have

$$
\begin{aligned}
\mathbf{E} 1_{A} & \left(\frac{R^{N}}{N^{3 / 2}}\right) f\left(\frac{\mathbf{J}^{N}-N \mathbf{b}}{N^{1 / 2}}\right) \\
& =\mathbf{E} \mathbf{P}\left(\frac{R^{N}}{N^{3 / 2}} \in A \mid \mathbf{J}^{N}\right) f\left(\frac{\mathbf{J}^{N}-N \mathbf{b}}{N^{1 / 2}}\right) \\
& =\mathbf{E} \mathbf{P}\left(\frac{\operatorname{Inv}\left(\mathbf{L}^{N}, \mathbf{J}^{N}\right)-e\left(\mathbf{L}^{N}, \mathbf{J}^{N}\right)}{N^{3 / 2}} \in A\right) f\left(\frac{\mathbf{J}^{N}-N \mathbf{b}}{N^{1 / 2}}\right) .
\end{aligned}
$$

By Skorokhod's representation theorem (see $[2,29])$ we may assume that $\frac{\mathbf{J}^{N}-N \mathbf{b}}{N^{1 / 2}} \stackrel{d}{\longrightarrow} \mathbf{X}$, $\mathbf{P}-$ a.s., where $\mathbf{X} \stackrel{d}{=} \mathcal{N}(\mathbf{0}, \Sigma)$. Then clearly $\frac{1}{N} \mathbf{J}^{N} \rightarrow \mathbf{b}, \mathbf{P}-$ a.s., and by the preceding lemma

$$
\mathbf{P}\left(\frac{\operatorname{Inv}\left(\mathbf{L}^{N}, \mathbf{J}^{N}\right)-e\left(\mathbf{L}^{N}, \mathbf{J}^{N}\right)}{N^{3 / 2}} \in A\right) \longrightarrow \mathcal{N}(0, v(\mathbf{a}, \mathbf{b}))(A), \mathbf{P}-\text { a.s. }
$$

Therefore,

$$
\mathbf{E} 1_{A}\left(\frac{R^{N}}{N^{3 / 2}}\right) f\left(\frac{\mathbf{J}^{N}-N \mathbf{b}}{N^{3 / 2}}\right) \longrightarrow \mathcal{N}(0, v(\mathbf{a}, \mathbf{b}))(A) \mathbf{E}(f(\mathbf{X}))
$$

Finally we need a result that enables us to treat the occurring quadratic functions of $\mathbf{J}^{N}$. For quadratic functions of asymptotically normal random vectors $\mathbf{X}^{N}$ one has the following elementary result.

Proposition 2.10. Assume there exists $\mathbf{b} \in \mathbf{R}^{m}$ and a positive semidefinite matrix $\Sigma \in$ $\mathbf{R}^{m \times m}$ of positive rank such that

$$
\frac{\mathbf{E}\left(\mathbf{X}^{N}\right)}{N} \longrightarrow \mathbf{b} \quad \text { and } \quad \frac{\mathbf{X}^{N}-N \mathbf{b}}{N^{1 / 2}} \stackrel{d}{\longrightarrow} \mathcal{N}(\mathbf{0}, \Sigma) .
$$


Let $M \in \mathbf{R}^{m \times m}, \mathbf{v} \in \mathbf{R}^{m}$, and consider the quadratic function $q(\mathbf{x}, \mathbf{v})=\mathbf{x} M \mathbf{x}^{t}+\mathbf{v} \mathbf{x}^{t}$. If we assume that $\mathbf{v}^{N}$ is a sequence with $\frac{1}{N} \mathbf{v}^{N} \rightarrow \mathbf{a}$ and let $\mathbf{w}=\mathbf{b}\left(M+M^{t}\right)+\mathbf{a}$, then

$$
\frac{q\left(\mathbf{X}^{N}, \mathbf{v}^{N}\right)-q\left(\mathbf{E}\left(\mathbf{X}^{N}\right), \mathbf{v}^{N}\right)}{N^{3 / 2}} \stackrel{d}{\longrightarrow} \mathcal{N}\left(0, \mathbf{w} \Sigma \mathbf{w}^{t}\right)
$$

If additionally $\frac{1}{N} \operatorname{Cov}\left(\mathbf{X}^{N}\right) \longrightarrow \Sigma$, then

$$
\frac{\mathbf{E}\left(q\left(\mathbf{X}^{N}, \mathbf{v}^{N}\right)\right)}{N^{2}} \longrightarrow q(\mathbf{b}, \mathbf{a})
$$

and

$$
\frac{\mathbf{E}\left(q\left(\mathbf{X}^{N}, \mathbf{v}^{N}\right)-q\left(\mathbf{E}\left(\mathbf{X}^{N}\right), \mathbf{v}^{N}\right)\right)}{N} \longrightarrow \sum_{i, j} M_{i, j} \Sigma_{i, j} .
$$

If furthermore $\mathbf{E}\left(\mathbf{X}_{i}^{N}-\mathbf{E}\left(\mathbf{X}_{i}^{N}\right)\right)^{4} / N^{3} \longrightarrow 0$ for all $i$, then

$$
\frac{\operatorname{Var}\left(q\left(\mathbf{X}^{N}, \mathbf{v}^{N}\right)\right)}{N^{3}} \longrightarrow \mathbf{w} \Sigma \mathbf{w}^{t}
$$

Combining Proposition 2.10 with Theorem 2.9 gives a complete picture of the asymptotic distributions of the random variables considered above.

Corollary 2.11. In the situation of Theorem 2.9 assume that additionally $\frac{1}{N} \mathbf{E}\left(\mathbf{J}^{N}\right) \rightarrow \mathbf{b}$. Let $b_{0}=b_{m+1}=0, J_{m+1}^{N}=0, C^{N}=\frac{1}{2} \sum_{i=1}^{m}\left(L_{i}^{N}+\mathbf{E}\left(J_{i+1}^{N}\right)-\mathbf{E}\left(J_{i}^{N}\right)\right) \mathbf{E}\left(J_{i}^{N}\right)$, and $D^{N}=\sum_{i=1}^{m} \mathbf{E}\left(J_{i}^{N}\right)\left(\mathbf{E}\left(J_{i}^{N}\right)+\sum_{k=1}^{i-1} L_{k}^{N}\right)$, and let $\mathbf{c}=\mathbf{c}(\mathbf{a}, \mathbf{b})$ be the vector with coordinates $c_{i}=a_{i}+b_{i+1}+b_{i-1}-2 b_{i}$ and $\mathbf{f}=\mathbf{f}(\mathbf{a}, \mathbf{b})$ be the vector with coordinates $f_{i}=a_{i}+b_{i+1}+$ $b_{i-1}+2 b_{i}+2 \sum_{\ell=1}^{i-1} a_{\ell}$. Then,

$$
\begin{gathered}
\frac{e\left(\mathbf{L}^{N}, \mathbf{J}^{N}\right)-C^{N}}{N^{3 / 2}} \stackrel{d}{\longrightarrow} \mathcal{N}\left(0, \frac{1}{4} \mathbf{c} \Sigma \mathbf{c}^{t}\right), \\
\frac{Y^{N}-C^{N}}{N^{3 / 2}} \stackrel{d}{\longrightarrow} \mathcal{N}\left(0, \frac{1}{4} \mathbf{c} \Sigma \mathbf{c}^{t}+v(\mathbf{a}, \mathbf{b})\right), \\
\frac{T^{N}-\left(C^{N}+D^{N}\right)}{N^{3 / 2}} \stackrel{d}{\longrightarrow} \mathcal{N}\left(0, \frac{1}{4} \mathbf{f} \Sigma \mathbf{f}^{t}+v(\mathbf{a}, \mathbf{b})\right) .
\end{gathered}
$$

Proof. The first assertion follows directly from Proposition 2.10. For the second assertion observe that by Theorem 2.9 the limiting distribution is the convolution of the normal distributions $R(a, b)$ and the limiting distribution in (12).

Finally, the variance of the rest-inversion part $R^{N}$ is as follows.

Lemma 2.12. In the situation of Theorem 2.9 assume that additionally $\frac{1}{N} \mathbf{E}\left(\mathbf{J}^{N}\right) \rightarrow \mathbf{b}$, and that $\frac{1}{N} \mathbf{J}^{N}$ is bounded. Then,

$$
\frac{\operatorname{Var}\left(R^{N}\right)}{N^{3}} \longrightarrow v(\mathbf{a}, \mathbf{b})
$$


Proof. We have $\mathbf{E}\left(\left(R^{N}\right)^{2} \mid \mathbf{J}^{N}\right)=\operatorname{Var}\left(\operatorname{Inv}\left(\mathbf{L}^{N}, \mathbf{J}^{N}\right)\right)$. Therefore

$$
\mathbf{E}\left(\left(R^{N}\right)^{2} \mid \mathbf{J}^{N}\right)=\frac{1}{12} \sum_{i=1}^{m}\left(L_{i}^{N}+J_{i+1}^{N}-J_{i}^{N}\right) J_{i}^{N}\left(L_{i}^{N}+J_{i+1}^{N}\right),
$$

and by our assumptions $\mathbf{E}\left(\left(R^{N}\right)^{2} \mid \mathbf{J}^{N}\right) / N^{3}$ converges boundedly to $v(\mathbf{a}, \mathbf{b})$. Hence we have $\mathbf{E}\left(\left(R^{N}\right)^{2}\right) / N^{3}=\mathbf{E}\left(\left(\left(R^{N}\right)^{2} \mid \mathbf{J}^{N}\right)\right) / N^{3} \longrightarrow v(\mathbf{a}, \mathbf{b})$.

\subsection{Unrestricted number of parts}

We first consider the total number $\tilde{T}(\mathbf{L})(q)$ of $\mathbf{L}$-admissible partitions. In this case clearly $A_{k-i+1}(k)$ (as defined in Proposition 2.4) has a binomial distribution with parameters $n=$ $L_{k}$ and $p=\frac{i}{k+1}$, and hence each $J_{i}$ can be represented as a sum of independent binomial variables. Furthermore, the covariance of $A_{k-i+1}(k)$ and $A_{k-j+1}(k)$ can be computed as

$$
\operatorname{Cov}\left(A_{k-i+1}(k), A_{k-j+1}(k)\right)=\frac{L_{k}}{k+1}\left(\min (i, j)-\frac{i j}{k+1}\right) .
$$

We therefore have

Lemma 2.13. Consider $\left(J_{1}, \ldots, J_{m}\right)$ as defined in Proposition 2.4. Then,

$$
\begin{aligned}
\mathbf{E}\left(J_{i}\right) & =i \sum_{k=i}^{m} \frac{L_{k}}{k+1}, \\
\operatorname{Var}\left(J_{i}\right) & =i \sum_{k=i}^{m} \frac{L_{k}}{k+1}-i^{2} \sum_{k=i}^{m} \frac{L_{k}}{(k+1)^{2}} \\
\operatorname{Cov}\left(J_{i}, J_{j}\right) & =\min (i, j) \sum_{k=\max (i, j)}^{m} \frac{L_{k}}{k+1}-i j \sum_{k=\max (i, j)}^{m} \frac{L_{k}}{(k+1)^{2}} .
\end{aligned}
$$

Moreover, straightforward computations lead to the exact expectation value of the random variable defined in (7).

Lemma 2.14. Consider the random variables defined in (7), and let $s_{i}=\sum_{k=i}^{m} \frac{L_{k}}{k+1}$, $t_{i}=\sum_{k=1}^{i-1} L_{k}$. Then, for $\mathbf{E}(T)=\mathbf{E}(Y)+\mathbf{E}(Q(\mathbf{L}, \mathbf{J}))$ we have

$$
\begin{aligned}
\mathbf{E}(Y) & =\frac{1}{2} \sum_{i=1}^{m} i s_{i}^{2}-\frac{1}{4} \sum_{i=1}^{m} \frac{i}{i+1} L_{i}, \\
\mathbf{E}(Q(\mathbf{L}, \mathbf{J})) & =\sum_{i=1}^{m} i^{2} s_{i}^{2}+\sum_{i=1}^{m} i s_{i} t_{i}+\sum_{i=1}^{m} \frac{i(i+2)}{6(i+1)} L_{i} .
\end{aligned}
$$

Theorem 2.15. Let $\frac{1}{N} \mathbf{L}^{N} \rightarrow \mathbf{a} \neq \mathbf{0}$. Then, $\frac{1}{N} \mathbf{E}\left(J_{i}^{N}\right) \rightarrow i \sum_{k=i}^{m} \frac{a_{k}}{k+1}$ for each $i$ and

$$
\frac{\mathbf{J}^{N}-\mathbf{E}\left(\mathbf{J}^{N}\right)}{N^{1 / 2}} \stackrel{d}{\longrightarrow} \mathcal{N}(\mathbf{0}, \Sigma)
$$


where

$$
\Sigma_{i, j}=\min (i, j) \sum_{k=\max (i, j)}^{m} \frac{a_{k}}{k+1}-i j \sum_{k=\max (i, j)}^{m} \frac{a_{k}}{(k+1)^{2}} .
$$

Proof. The first assertion is clear. For the second assertion let $\mathbf{B}_{\mathbf{L}^{N}}$ denote the underlying total occupancy statistic. In $\S 2.2$ it was shown that the components $\mathbf{B}(i)^{N}$ are indendent multinomial distributions with parameters $L_{i}^{N}$ and $\mathbf{u}(i)$, where $u(i)_{0}=\ldots=u(i)_{i}=$ $\frac{1}{i+1}$, and covariances $L_{i}^{N} \Sigma(i), \Sigma(i)=\operatorname{diag}(\mathbf{u}(i))-\mathbf{u}^{t}(i) \mathbf{u}(i)$. By Theorem 2.3, and since $\frac{1}{N} L_{i}^{N} \rightarrow a_{i}$, we have

$$
\frac{\mathbf{B}_{\mathbf{L}^{N}}-\mathbf{E}\left(\mathbf{B}_{\mathbf{L}^{N}}\right)}{N^{1 / 2}} \stackrel{d}{\longrightarrow}\left(\mathcal{N}\left(\mathbf{0}, a_{1} \Sigma(1)\right), \ldots, \mathcal{N}\left(\mathbf{0}, a_{m} \Sigma(m)\right)\right)
$$

where the components on the right-hand side are independent. Since $\mathbf{J}^{N}$ is a linear image of $\mathbf{B}_{\mathbf{L}}^{N}$ it is clear that $\mathbf{J}^{N}$ is asymptotically normal. The assertion about the covariance matrix is obvious.

It is clear from Corollary 2.11 that under the conditions of Theorem 2.15 the number of admissible partitions is asymptotically normal, with expectation of order $N^{2}$ and variance of order $N^{3}$. The final condition of Proposition 2.10 is fulfilled since the coordinates of the $\mathbf{B}(i)$ are binomially distributed and therefore have central fourth moments of order $L_{i}^{2}$. The boundedness condition of Lemma 2.12 is fulfilled since $J_{i}^{N} \leqslant \sum_{k=1}^{m} L_{k}^{N}$.

\subsection{The central restricted case}

We consider the "central region" as discussed in $\S 2.2 .3$ and let $s_{N}=\left\lfloor\mathbf{E}\left(S_{\mathbf{L}^{N}}\right)\right\rfloor=$ $\left\lfloor\frac{1}{2} \sum_{i=1}^{m} i L_{i}\right\rfloor$. It is clear from the above that the underlying occupancy distribution $\mathbf{B}_{\mathbf{L}^{N}}$ is the conditional distribution

$$
\mathbf{B}_{\mathbf{L}^{N}}=\left(\mathbf{Y}(1)^{N}, \ldots, \mathbf{Y}(m)^{N}\right) \mid \sum_{k=1}^{m} \sum_{i=0}^{k} Y_{i}(k)^{N}=s_{N},
$$

where $\mathbf{Y}(1)^{N}, \ldots, \mathbf{Y}(m)^{N}$ are independent random vectors, each $\mathbf{Y}(k)^{N}$ is multinomial with parameters $L_{k}^{N}$ and $p_{0}=\ldots=p_{k}=\frac{1}{k+1}$. This conditioning has the following effect on the asymptotic distribution.

Theorem 2.16. Let $m>1, s_{N}=\left\lfloor\mathbf{E}\left(S_{\mathbf{L}^{N}}\right)\right\rfloor$ and $\mathbf{u}=(\mathbf{u}(1), \mathbf{u}(2), \ldots, \mathbf{u}(m))$ with $\mathbf{u}(k)=$ $\left(\frac{1}{k+1}, \ldots, \frac{1}{k+1}\right)$. Assume that $\frac{1}{N} \mathbf{L}^{N} \longrightarrow \mathbf{a} \neq \mathbf{0}$ and let $\sigma^{2}(\mathbf{a})=\frac{1}{12} \sum_{k=1}^{m} k(k+2) a_{k}$. Then,

$$
\frac{\mathbf{B}^{N}-N \mathbf{u}}{\sqrt{N}} \stackrel{d}{\longrightarrow} \mathcal{N}(0, \Sigma)
$$

where

$$
\begin{array}{r}
\Sigma_{i, j}(k, \ell)=a_{k} \delta_{k, \ell}\left(\frac{1}{k+1} \delta_{i, j}-\frac{1}{(k+1)^{2}}\right) \\
-a_{k} a_{\ell} \frac{(k-2 i)(\ell-2 j)}{4(k+1)(\ell+1) \sigma^{2}(\mathbf{a})} .
\end{array}
$$


Proof. We give the proof for the one component case $\mathbf{L}^{N}=\left(L_{1}^{N}, \ldots, L_{m-1}^{N}, L_{m}^{N}\right)=$ $(0, \ldots, 0, N)$, the generalization is straightforward. Let $\mathbf{B}^{N}:=\mathbf{B}(m)^{N}$, and $X_{1}, \ldots, X_{N}$ be i.i.d. random variables uniform on $\{0, \ldots, m\}$. Let $S_{N}:=\sum_{i=1}^{N} X_{i}, \mu=m / 2$, $\sigma^{2}=\frac{1}{12} m(m+2)$. The probability generating function of $\mathbf{B}^{N}$ is

$$
\mathbf{E}\left(\prod_{i=0}^{m} t_{i}^{B_{i}^{N}}\right)=\left[x^{s_{N}}\right]\left(t_{0}+t_{1} x+\ldots+t_{m} x^{m}\right)^{N} /\left((m+1)^{n} \mathbf{P}\left(S_{N}=s_{N}\right)\right) .
$$

Hence the joint distribution is given by

$$
\mathbf{P}\left(B_{0}^{N}=k_{0}, \ldots, B_{m}^{N}=k_{m}\right)=\left(\begin{array}{c}
N \\
k_{0}, \ldots, k_{m}
\end{array}\right) /\left((m+1)^{N} \mathbf{P}\left(S_{N}=s_{N}\right)\right)
$$

with the constraints that $\sum_{i=0}^{m} k_{i}=N$ and $\sum_{i=1}^{n} i k_{i}=s_{N}$. Since there are two linearly independent linear constraints on the values of $\mathbf{B}^{N}$ we expect a $(m-1)$-dimensional limiting distribution. Let $x_{0}, \ldots, x_{m}$ be real numbers with $\sum_{i=0}^{m} x_{i}=0$ and $\sum_{i=0}^{m} i x_{i}=0$, and let $k_{i}=\frac{N}{i+1}+\sqrt{N} x_{i}$. By Stirling's approximation for the factorials for the numerator and the local limit theorem for lattice distributions for the denominator we see

$$
(\sqrt{N})^{m-1} \mathbf{P}\left(\mathbf{B}^{N}=\mathbf{k}\right) \longrightarrow \frac{1}{\sqrt{(2 \pi)^{m-1}}} \sqrt{(m+1)^{m+1} \sigma^{2}} e^{-\frac{m+1}{2}\left(x_{0}^{2}+\ldots+x_{m}^{2}\right)} .
$$

A check that the expression on the right-hand side (considered as a function of $x_{2}, \ldots, x_{m}$, say) is the marginal density of $(\mathcal{N}(0, \Sigma))_{2, \ldots, m}$ with $\Sigma$ as in 18 concludes the proof.

For the convergence of moments we have

Proposition 2.17. Under the conditions of Theorem 2.16

$$
\frac{\mathbf{E}\left(\mathbf{B}(k)^{N}\right)}{N} \longrightarrow a_{k} \mathbf{u}(k) \quad \text { and } \quad \frac{\operatorname{Cov}\left(B_{i}(k)^{N}, B_{j}(\ell)^{N}\right)}{N} \stackrel{d}{\longrightarrow} \Sigma_{i, j}(k, \ell)
$$

Furthermore, $\mathbf{E}\left(\mathbf{B}_{i}(k)^{N}-\mathbf{E}\left(\mathbf{B}_{i}(k)^{N}\right)\right)^{4} / N^{3} \longrightarrow 0$.

Proof. Again we restrict the exposition to the one component case and use the same notation as in the proof of Theorem 2.16. From the generating function given there we get

$$
\begin{aligned}
\mathbf{E}\left(B_{i}^{N}\right) & =\frac{N}{m+1} \frac{\mathbf{P}\left(S_{N-1}=s_{N}-i\right)}{\mathbf{P}\left(S_{N}=s_{N}\right)}, \\
\mathbf{E}\left(B_{i}^{N}\right)^{2} & =\mathbf{E}\left(B_{i}^{N}\right)+\frac{N(N-1)}{(m+1)^{2}} \frac{\mathbf{P}\left(S_{N-2}=s_{N}-2 i\right)}{\mathbf{P}\left(S_{N}=s_{N}\right)}, \\
\mathbf{E}\left(B_{i}^{N} B_{j}^{N}\right) & =\frac{N(N-1)}{(m+1)^{2}} \frac{\mathbf{P}\left(S_{N-2}=s_{N}-i-j\right)}{\mathbf{P}\left(S_{N}=s_{N}\right)} .
\end{aligned}
$$

By the local central limit theorem for lattice distributions [14, Corollary VIII.3] we have

$$
\mathbf{P}\left(S_{N}=k\right)=\frac{1}{\sqrt{2 \pi N}} e^{-\frac{(k-N \mu)^{2}}{2 N \sigma^{2}}}\left(1+O\left(N^{-1 / 2}\right)\right)
$$


if $\left|\frac{(k-N \mu)^{2}}{2 N \sigma^{2}}\right|<C$. Applying this to the numerator and denominator shows that the quotients $q_{r}(N):=\frac{\mathbf{P}\left(S_{N-r}=s_{N}-r i\right)}{\mathbf{P}\left(S_{N}=s_{N}\right)}$ are asymptotically of the form $q_{r}(N)=1-\frac{c_{r}}{2 \sigma^{2} N}+O\left(N^{-3 / 2}\right)$ where $c_{r}=r^{2}(m / 2-i)^{2}$. Now, the asympotic assertion about the expectation follows immediately from the local limit theorem, applied to numerator and denominator in 19. The asymptotic assertion about the variance/covariance follows from the formulæ above using the asymptotic form of $q_{1}(N), q_{2}(N)$. Concerning the asserted convergence of the central fourth moment, note that the $r-t h$ factorial moment of $B_{i}^{N}$ is

$$
\begin{aligned}
& \mathbf{E}\left(B_{i}^{N}\left(B_{i}^{N}-1\right) \cdots\left(B_{i}^{N}-r+1\right)\right) \\
& =\frac{N(N-1) \cdots(N-r+1)}{(m+1)^{r}} \frac{\mathbf{P}\left(S_{N-r}=s_{N}-r i\right)}{\mathbf{P}\left(S_{N}=s_{N}\right)}
\end{aligned}
$$

After expressing the central fourth moment as a linear combination of factorial moments, plugging in the asymptotic expressions for the $q_{r}(N)$, and noting that $c_{4}+6 c_{2}-4 c_{3}-4 c_{1}=$ 0 , one obtains

$$
\mathbf{E}\left(B_{i}^{N}-\mathbf{E}\left(B_{i}^{N}\right)^{4}\right)=N^{3}\left(c_{4}+6 c_{2}-4 c_{3}-4 c_{1}\right)+O\left(N^{5 / 2}\right)=O\left(N^{5 / 2}\right) .
$$

Remark 2.18. Let $\frac{1}{N} \mathbf{L}^{N} \rightarrow \mathbf{a} \neq \mathbf{0}$. A comparison to the unrestricted case, discussed in $\S 2.4$, shows that asymptotically the underlying total occupancy distributions are quite similar. They concentrate around the same expectations. In the unrestricted case the components of the limiting distribution $\frac{1}{\sqrt{N}}\left(\mathbf{B}_{\mathbf{L}^{N}}-\mathbf{u}\right) \stackrel{d}{\longrightarrow} Z$ are independent normal vectors with

$$
\operatorname{Cov}(Z(k))=a_{k}\left(\operatorname{diag}(\mathbf{u}(k))-\mathbf{u}(k)^{t} \mathbf{u}(k)\right) .
$$

The components stay normal in the central restricted case, but the restriction causes an additional negative correlation

$$
\operatorname{Cov}\left(Z(k)_{i}, Z(\ell)_{j}\right)=-\frac{a_{k} a_{\ell}(2 k-i)(2 \ell-j)}{4(k+1)(l+1) \sigma^{2}(\mathbf{a})}
$$

between the components. This in turn forces the elements of the asymptotic covariance $\Sigma$ of $\mathbf{J}^{N}$ to be smaller than in the unrestricted case, we compute

$$
\Sigma_{i, j, \text { restricted }}=\Sigma_{i, j, \text { unrestricted }}-\frac{i j}{2 \sigma^{2}(\mathbf{a})} c(i) c(j)
$$

with $c(i)=\sum_{k=i}^{m} \frac{k+1-i}{k+1} a_{k}$.

Since $\mathbf{J}^{N}$ is a linear image of $\mathbf{B}^{N}$ its distribution is also asymptotically normal and it is clear from Theorem 2.16 and Corollary 2.11 that $T^{N}$ is asymptotically normal and the preceding results show that the expectation resp. variance of $T^{N}$ are of $N^{2}$ resp. $N^{3}$, but the variance in the restricted case will (on the $N^{3}$ scale) be smaller than in the unrestricted case.

Remark 2.19. The cases $\frac{1}{N} S_{\mathbf{L}^{N}} \rightarrow a^{\prime} \neq a:=\frac{1}{2} \sum_{k=1}^{m} k a_{k}$ can be treated by standard large deviation techniques. Again we may safely expect to find normality of the asymptotic distributions. 


\section{Interpretation in terms of fusion and Demazure modules}

In the sequel we will consider characters of fusion modules of the current algebra defined by Feigin an Loktev in [11], and Demazure modules of the corresponding affine algebra. As mentioned in the introduction those characters can be interpreted as Hilbert series whose coefficients encode dimensions of so-called weight spaces. We will be mostly concerned with the current algebra $\mathfrak{s l}_{2} \otimes \mathbf{C}[t]$ and the closely related affine Kac-Moody algebra $\widehat{\mathfrak{s l}}_{2}$. For further reading we refer the reader to $[8,9,10,15,19]$, and for general facts about current and affine Kac-Moody algebras and their representation theory to [7, 18].

\subsection{Fusion modules}

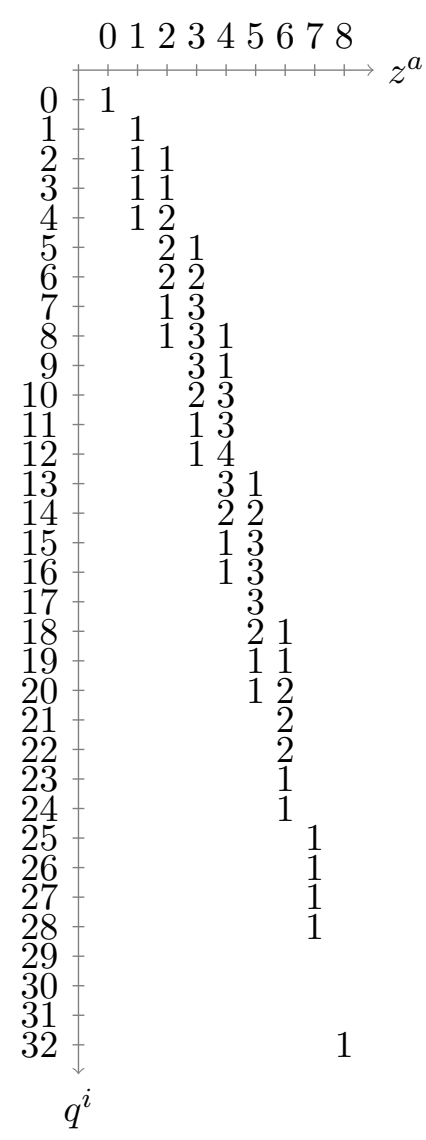

Figure 1: Plot of the character of the fusion module $\mathcal{F}(0,4)=\left(\mathbf{C}^{3}\right)^{* 4}$, that is $\chi(\mathcal{F}(0,4))(z, q)=\sum_{a \in \mathbf{Z}} z^{a} \cdot \sum_{j_{1}+j_{2}=a} q^{j_{1}^{2}+j_{2}^{2}}\left[\begin{array}{c}4 \\ j_{2}\end{array}\right]_{q}\left[\begin{array}{c}j_{2} \\ j_{1}\end{array}\right]_{q}$.

Let us consider fusion modules for the current algebra $\mathfrak{s l}_{2} \otimes \mathbf{C}[t][9,10]$ :

$$
\mathcal{F}\left(L_{1}, \ldots, L_{m}\right)=\left(\mathbf{C}^{2}\right)^{* L_{1}} * \cdots *\left(\mathbf{C}^{m+1}\right)^{* L_{m}} .
$$


Feigin and Feigin prove in [9, Theorem 5.1] that, in terms of the $q$-supernomial $\tilde{T}(\mathbf{L}, a)(q)$ from (1), its graded character is given as

$$
\chi\left(\mathcal{F}\left(L_{1}, \ldots, L_{m}\right)\right)(z, q)=\sum_{a \in \mathbf{Z}} z^{a} \cdot \tilde{T}(\mathbf{L}, a)(q)
$$

Here $q$ refers to the grading as introduced by Feigin and Loktev [11]. The specialization at $q=1$ of the graded character $\chi\left(\mathcal{F}\left(L_{1}, \ldots, L_{m}\right)\right)(z, q)$ equals the character of the tensor product $\left(\mathbf{C}^{2}\right)^{\otimes L_{1}} \otimes \cdots \otimes\left(\mathbf{C}^{m+1}\right)^{\otimes L_{m}}$ of irreducible representations of $\mathfrak{s l}_{2}$, i.e. the variable $z$ associates with the grading by the simple root $\alpha_{1}$ of $\mathfrak{s l}_{2}$. Since those characters multiply, i.e. $\chi(V \otimes W)=\chi(V) \cdot \chi(W)$, the associated probability distributions convolute and asymptotic considerations reduce to the central limit theorem for sums of i.i.d. random variables. Therefore, the specializations at $q=1$ are well understood from a statistical point of view and have been analyzed in great detail, e.g. [31]. Much less studied is the so-called basic specialization ${ }^{2}$ of those characters, i.e. their evaluation at $z=1$. Now, we have

Theorem 3.1. Consider a sequence $\left(\mathbf{C}^{2}\right)^{* L_{1}^{N}} * \cdots *\left(\mathbf{C}^{m+1}\right)^{* L_{m}^{N}}$ of fusion modules of the current algebra $\mathfrak{s l}_{2} \otimes \mathbf{C}[t]$. If $\frac{1}{N}\left(L_{1}^{N}, \ldots, L_{m}^{N}\right) \rightarrow \mathbf{a} \neq 0$, then the sequence of basic specializations, that is $\sum_{a \in \mathbf{Z}} \tilde{T}\left(\left(L_{1}^{N}, \ldots, L_{m}^{N}\right), a\right)(q)$, behaves asymptotically normal with individual means

$$
\begin{aligned}
\mu_{\left(L_{1}^{N}, \ldots, L_{m}^{N}\right)}=\sum_{i=1}^{m} & {\left[\left(\frac{i}{2}+i^{2}\right)\left(\sum_{k=i}^{m} \frac{L_{k}^{N}}{k+1}\right)^{2}\right.} \\
+ & \left.i\left(\sum_{k=i}^{m} \frac{L_{k}^{N}}{k+1}\right)\left(\sum_{k=1}^{i-1} L_{k}^{N}\right)+\left(\frac{4 i(i+2)-6 i}{24(i+1)}\right) L_{i}^{N}\right],
\end{aligned}
$$

and variance, as $N \rightarrow \infty$,

$$
\frac{1}{N^{3}} \sigma_{\left(L_{1}^{N}, \ldots, L_{m}^{N}\right)}^{2} \rightarrow \frac{1}{4} \mathbf{f} \Sigma \mathbf{f}^{t}+v(\mathbf{a}, \mathbf{b})
$$

Here, the vectors $\mathbf{a}, \mathbf{b}, \mathbf{f}$, the function $v$, and the matrix $\Sigma$ are given by

$$
\begin{aligned}
& \mathbf{a}=\left(a_{1}, \ldots, a_{m}\right)=\lim _{N \rightarrow \infty} \frac{1}{N}\left(L_{1}^{N}, \ldots, L_{m}^{N}\right), \\
& b_{i}=i \sum_{\ell=i}^{m} \frac{a_{\ell}}{\ell+1}, \\
& f_{i}=a_{i}+b_{i+1}+b_{i-1}+2 b_{i}+2 \sum_{\ell=1}^{i-1} a_{\ell},
\end{aligned}
$$

\footnotetext{
${ }^{2}$ We borrow this terminology from $\operatorname{Kac}[18, \S 1.5,10.8,12.2]$ who analyzed this kind of specialization for characters of integrable highest weight modules $V(\Lambda)$, and obtained Macdonald's identities for Dedekind's $\eta$-function $[18, \S 12.2]$.
} 


$$
\begin{aligned}
v(\mathbf{a}, \mathbf{b}) & =\frac{1}{12} \sum_{i=1}^{m}\left(a_{i}+b_{i+1}-b_{i}\right) b_{i}\left(a_{i}+b_{i+1}\right)\left(\text { where } b_{m+1}=0\right), \\
\Sigma_{i, j} & =\min (i, j) \sum_{k=\max (i, j)}^{m} \frac{a_{k}}{k+1}-i j \sum_{k=\max (i, j)}^{m} \frac{a_{k}}{(k+1)^{2}} .
\end{aligned}
$$

Proof. Recall from (7) that the basic specialization is the generating function for the distribution of the random variable $T^{N}=Q\left(\mathbf{L}^{N}, \mathbf{J}^{N}\right)+Y^{N}$ from (8). As such the mean $\mu_{\left(L_{1}^{N}, \ldots, L_{m}^{N}\right)}$ is equal to $\mathbf{E}\left(T^{N}\right)$ as described in Lemma 2.14. The assertions about the asymptotic normality and the variance are recollections from Theorem 2.9 and Corollary 2.11 which apply in the present situation due to Theorem 2.15 .

The central string functions in fusion modules behave quite similar to the basic specialization. The following is just a recollection of the results in $\S 2.5$, and in particular the observations noted in Remark 2.18.

Theorem 3.2. Consider a sequence $\left(\mathbf{C}^{2}\right)^{* L_{1}^{N}} * \cdots *\left(\mathbf{C}^{m+1}\right)^{* L_{m}^{N}}$ of fusion modules of the current algebra $\mathfrak{s l}_{2} \otimes \mathbf{C}[t]$. If $\frac{1}{N}\left(L_{1}^{N}, \ldots, L_{m}^{N}\right) \rightarrow \mathbf{a} \neq 0$, then the sequence of central string functions, that is $\tilde{T}\left(\left(L_{1}^{N}, \ldots, L_{m}^{N}\right), s_{N}\right)(q)$ with $s_{N}=\left\lfloor\frac{1}{2} \sum_{i=1}^{m} i L_{i}^{N}\right\rfloor$, behaves asymptotically normal with asymptotic mean

$$
\begin{aligned}
& \frac{1}{N^{2}} \mu_{\left(L_{1}^{N}, \ldots, L_{m}^{N}\right), s_{N}} \rightarrow \\
& \quad \sum_{i=1}^{m}\left[\left(\frac{i}{2}+i^{2}\right)\left(\sum_{k=i}^{m} \frac{a_{k}}{k+1}\right)^{2}+i\left(\sum_{k=i}^{m} \frac{a_{k}}{k+1}\right)\left(\sum_{k=1}^{i-1} a_{k}\right)\right],
\end{aligned}
$$

and asymptotic variance

$$
\frac{1}{N^{3}} \sigma_{\left(L_{1}^{N}, \ldots, L_{m}^{N}\right), s_{N}}^{2} \rightarrow \frac{1}{4} \mathbf{f} \Sigma \mathbf{f}^{t}+v(\mathbf{a}, \mathbf{b}) .
$$

Here, the vectors $\mathbf{a}, \mathbf{b}, \mathbf{f}$ and the function $v$ are as in Theorem 3.1. The matrix $\Sigma$ is given as

$$
\Sigma_{i, j}=-\frac{i j}{2 \sigma^{2}(\mathbf{a})} c(i) c(j)+\min (i, j) \sum_{k=\max (i, j)}^{m} \frac{a_{k}}{k+1}-i j \sum_{k=\max (i, j)}^{m} \frac{a_{k}}{(k+1)^{2}}
$$

where $\sigma^{2}(\mathbf{a})=\frac{1}{12} \sum_{k=1}^{m} k(k+2) a_{k}$, and $c(i)=\sum_{k=i}^{m} \frac{k+1-i}{k+1} a_{k}$.

The following corresponding local central limit theorems should hold.

Conjecture 3.3. In the notation of Theorem 3.1 let $X_{\mathcal{F}_{N}}$ denote a random variable with probability generating function the normalized basic specialization of the fusion module $\mathcal{F}_{N}=\mathcal{F}\left(L_{1}^{N}, \ldots, L_{m}^{N}\right)$. Denote its mean $\mu_{N}=\mu_{\left(L_{1}^{N}, \ldots, L_{m}^{N}\right)}$ and variance $\sigma_{N}^{2}=\sigma_{\left(L_{1}^{N}, \ldots, L_{m}^{N}\right)}^{2}$. Then, uniformly in $k$ as $N \rightarrow \infty$,

$$
\sqrt{2 \pi} \sigma_{N} \cdot \mathbf{P}\left(X_{\mathcal{F}_{N}}=k\right)=e^{-\left(k-\mu_{N}\right) / 2 \sigma_{N}^{2}}+o(1) .
$$

Here, $\sigma_{N}^{2}$ can be replaced by the explicit expression $N^{3}\left(\frac{1}{4} \mathbf{f} \Sigma \mathbf{f}^{t}+v(\mathbf{a}, \mathbf{b})\right)$ from (25). In particular, the dimension of the $\mathfrak{s l}_{2}$ submodule in $\mathcal{F}_{N}$ of degree $k$ grows as (28). 
Conjecture 3.4. In the notation of Theorem 3.2 let $S_{N}$ be a random variable with probability generating function the normalized central string function

$$
\mathbf{E}\left(q^{S_{N}}\right)=q^{-\frac{1}{2} l_{1}^{N} l_{m}^{N}} \tilde{T}\left(\mathbf{L}^{N}, s_{N}\right),
$$

where $l_{1}^{N}=\sum L_{i}^{N}$ and $l_{m}^{N}=\sum i L_{i}^{N}$. Let

$$
\begin{aligned}
\mu & =\lim _{N \rightarrow \infty} \frac{1}{N^{2}}\left(\mu_{\left(L_{1}^{N}, \ldots, L_{m}^{N}\right), s_{N}}-\frac{1}{2} l_{1}^{N} l_{m}^{N}\right), \\
\sigma^{2} & =\lim _{N \rightarrow \infty} \frac{1}{N^{3}}\left(\sigma_{\left(L_{1}^{N}, \ldots, L_{m}^{N}\right), s_{N}}^{2}\right) .
\end{aligned}
$$

Then, uniformly in $k$ as $N \rightarrow \infty$,

$$
\sqrt{2 \pi} \sigma \cdot \mathbf{P}\left(S_{N}=k\right)=e^{-(k-\mu) / 2 \sigma^{2}}+o(1)
$$

In particular, the dimension of the weight space with coordinates $\frac{1}{2} l_{m}^{N} \alpha_{1}$ and $-k \delta$ grows as (29).

Complemented by a result on the asymptotic normality of the basic specialization of graded tensors of the type $A$ standard representation (see [4]) we have a central limit theorem for a serious class of graded tensors. We will conclude with a general conjecture on the fusion modules of symmetric power representations for the current algebra $\mathfrak{s l}_{r} \otimes \mathbf{C}[t]$ in $\S 4$.

\subsection{Demazure modules}

It is well-known that Demazure modules $V_{w}(\Lambda)$ associated to $\widehat{\mathfrak{s l}}_{2}$ carry a $\mathfrak{s l}_{2} \otimes \mathbf{C}[t]$-module structure and as such are special instances of fusion modules (see e.g. [8, §1.5.1] or [15, $\S 3.5])$. To be precise, there are isomorphisms of $\mathfrak{s l}_{2} \otimes \mathbf{C}[t]$-modules

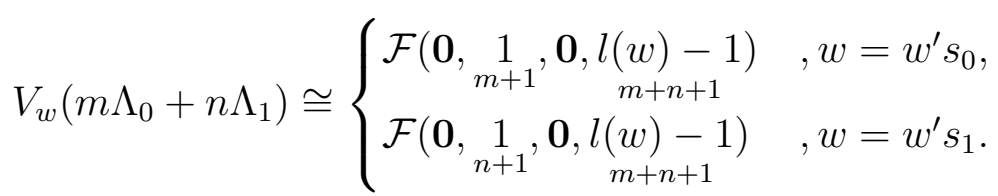

Here, we write $l(w)$ for the length of a reduced decomposition of $w \in W^{\text {aff }}$ in the affine Weyl group of $\widehat{\mathfrak{s l}}_{2}$. Note that the elements of $W^{\text {aff }}$ can be expressed as the following products of the simple reflections $s_{0}$ (corresponding to the imaginary root) and $s_{1}$ (corresponding to the simple root $\alpha_{1}$ ):

$$
\left(s_{1} s_{0}\right)^{N} s_{1}, N \geqslant 0 \text { and }\left(s_{0} s_{1}\right)^{N},\left(s_{1} s_{0}\right)^{N}, s_{0}\left(s_{1} s_{0}\right)^{N-1}, N>0 .
$$

The characters of Demazure modules can be identified as special instances of fusion modules through a series of translations (multiplication by $z^{a}$ ), reflections (evaluation at the reciprocal $1 / q$ ), and rotations (evaluation at mixed monomials $z q^{i}$ ), respectively. 
Proposition 3.5. Consider the Demazure module $V_{w}=V_{w}\left(m \Lambda_{0}+n \Lambda_{1}\right)$ of $\widehat{\mathfrak{s l}}_{2}$ of fixed highest weight $\Lambda=m \Lambda_{0}+n \Lambda_{1}$ and recall the character formula for fusion modules of the current algebra $\mathfrak{s l}_{2} \otimes \mathbf{C}[t](23)$. The character $\chi\left(V_{w}\right)(z, q)$, written in the coordinates $z=e^{-\alpha_{1}}$ and $q=e^{-\delta}\left(\alpha_{1}\right.$ is the simple root of $\mathfrak{s l}_{2}, \delta$ denotes the imaginary root), is given by

$$
\begin{aligned}
& \text { For the trivial element } w=\mathbf{1} \text { one has } \chi\left(V_{\mathbf{1}}\right)(z, q)=e^{\Lambda} \text {. } \\
& \text { For } w=\left(s_{1} s_{0}\right)^{N} s_{1}, N \geqslant 0 \text { one has } \\
& e^{-\Lambda} \chi\left(V_{w}\right)(z, q)= \\
& z^{-(n+m) N-n / 2} q^{N^{2} m+N(N+1) n} \chi\left(\mathcal{F}\left(0, \mathbf{L}_{w}\right)\left(z q^{-2 N-1}, q\right)\right. \\
& \text { where } \mathbf{L}_{w}=\left(L_{1}, \ldots, L_{m+n}\right)=(\mathbf{0}, \underset{n}{1}, \mathbf{0}, \underset{m+n}{2 N}) \text {. } \\
& \text { For } w=\left(s_{0} s_{1}\right)^{N}, N \geqslant 0 \text { one has } \\
& e^{-\Lambda} \chi\left(V_{w}\right)(z, q)= \\
& z^{-(n+m) N-n / 2} q^{N^{2} m+N(N+1) n} \chi\left(\mathcal{F}\left(0, \mathbf{L}_{w}\right)\left(z q^{-2 N-1}, q\right)\right. \\
& \text { where } \mathbf{L}_{w}=\left(L_{1}, \ldots, L_{m+n}\right)=(\mathbf{0}, \underset{n}{1,}, \underset{m+n}{2 N-1}) \text {. } \\
& \text { For } w=\left(s_{1} s_{0}\right)^{N}, N>0 \text { one has } \\
& e^{-\Lambda} \chi\left(V_{w}\right)(z, q)= \\
& z^{-(n+m) N+n / 2} q^{N^{2} m+N(N-1) n} \chi\left(\mathcal{F}\left(0, \mathbf{L}_{w}\right)\left(z q^{-2 N}, q\right)\right. \\
& \text { where } \mathbf{L}_{w}=\left(L_{1}, \ldots, L_{m+n}\right)=(\mathbf{0}, \underset{m}{1}, \mathbf{0}, \underset{m+n}{2 N-1}) \text {. } \\
& \text { For } w=s_{0}\left(s_{1} s_{0}\right)^{N-1}, N>0 \text { one has } \\
& e^{-\Lambda} \chi\left(V_{w}\right)(z, q)= \\
& z^{-(n+m) N+n / 2} q^{N^{2} m+N(N-1) n} \chi\left(\mathcal{F}\left(0, \mathbf{L}_{w}\right)\left(z q^{-2 N}, q\right)\right. \\
& \text { where } \mathbf{L}_{w}=\left(L_{1}, \ldots, L_{m+n}\right)=(\mathbf{0}, \underset{m}{1}, \mathbf{0}, \underset{m+n}{2 N-2}) \text {. }
\end{aligned}
$$

The sum of the entries in $\mathbf{L}_{w}$ represents the length $l(w)$ of the Weyl group element $w$. When either $n$ or $m$ equals 0 , then $\mathbf{L}_{w}=(\mathbf{0}, l(w))$.

Proof. Feigin [10, (11)] denotes an integrable highest weight representation by $L_{i, k}=$ $\mathcal{U}\left(\widehat{\mathfrak{s l}}_{2}\right) \cdot v_{i, k}$ with highest weight vector $v_{i, k}$ such that $c \cdot v_{i, k}=k v_{i, k}, h_{0} \cdot v_{i, k}=i v_{i, k}$, and $d \cdot v_{i, k}=0$. In our notation, the canonical central element is $c=\alpha_{0}^{\vee}+\alpha_{1}^{\vee}$, the coroot is $h_{0}=\alpha_{0}^{\vee}$, and the scaling element $d$ is given by $\alpha_{0}(d)=1$ and $\alpha_{1}(d)=0$. Therefore, by comparison of the highest weight vector we have $L_{i, k}=V\left((k-i) \Lambda_{0}+i \Lambda_{1}\right)$. The bigrading is chosen according to the action of $h_{0}$ and $d$, and consequently, the character is denoted in the monomials $e^{\alpha_{0}}=e^{\delta-\alpha_{1}}$ and $e^{-\delta}$, respectively. By [10, Corollary 3.1] each such module $L_{i, k}$ can be constructed as an inductive limit of fusion products, that is $L_{i, k}=\mathbf{C}^{i+1} *\left(\mathbf{C}^{k+1}\right)^{2 \infty}$. Each fusion product can be identified with the corresponding Demazure module $V_{w}\left((k-i) \Lambda_{0}+i \Lambda_{1}\right)$ by comparing the weights of the extremal weight vectors described in $[10, \S 1]$. Now apply the character formula [9, Theorem 5.1], noting that $e^{\alpha_{0}}=z q^{-1}$. 
We can now compare our findings to established results in the literature $[4,5,3,17]$.

\subsubsection{The unrestricted one component case}

Consider $\mathbf{L}=(0, \ldots, 0, N) \in \mathbf{Z}_{+}^{m}$ in which case the distribution of $Y^{N}$ has been investigated as a generalized Galois numbers by Bliem and Kousidis [4] and later on by Janson [17]. They studied the random variables $Y^{N}$ with probability generating function

$$
\mathbf{E}\left(q^{Y^{N}}\right)=\frac{1}{(m+1)^{N}} \sum_{\substack{\left(k_{0}, \ldots, k_{m}\right) \in \mathbf{N}_{0}^{m+1} \\
k_{0}+\cdots+k_{m}=N}}\left[\begin{array}{c}
N \\
k_{0}, \ldots, k_{m}
\end{array}\right]_{q}
$$

Note that if we let $B_{0}=L_{m}-J_{m}, B_{1}=J_{m}-J_{m-1}, \ldots, B_{m-1}=J_{2}-J_{1}, B_{m}=J_{1}$ it becomes evident that this distribution coincides with the distribution of $Y^{N}$ in the one component case. Now, Bliem and Kousidis showed

Theorem 3.6 ([4, Theorem 3.5]). Consider the random variables $Y^{N}$ defined through (36). Then,

$$
\begin{aligned}
\mathbf{E}\left(Y^{N}\right) & =\frac{1}{4} \frac{m}{m+1} N(N-1), \\
\operatorname{Var}\left(Y^{N}\right) & =\frac{1}{72} \frac{(m+1)^{2}-1}{(m+1)^{2}} N(N-1)(2 N+5), \\
\text { and } \quad \frac{Y^{N}-\mathbf{E}\left(Y^{N}\right)}{N^{3 / 2}} & \stackrel{d}{\longrightarrow} \mathcal{N}\left(0, \frac{1}{36} \frac{(m+1)^{2}-1}{(m+1)^{2}}\right) .
\end{aligned}
$$

Janson derived the same result in a variety of ways, proved a corresponding local central limit theorem, and gave different interpretations of the distribution of $Y^{N}$. He also showed joint convergence of $Y^{N}$ and $\mathbf{B}^{N}$ :

Theorem 3.7 ([17, Theorem 2.4]). Let $\mathbf{B}^{N}, Y^{N}$ be as above. Then,

$$
\begin{aligned}
& \left(\frac{Y^{N}-\mathbf{E}\left(Y^{N}\right)}{N^{3 / 2}}, \frac{\mathbf{B}^{N}-\mathbf{E}\left(\mathbf{B}^{N}\right)}{N^{1 / 2}}\right) \\
& \quad \stackrel{d}{\longrightarrow}\left(\mathcal{N}\left(0, \frac{1}{36} \frac{(m+1)^{2}-1}{(m+1)^{2}}\right), \mathcal{N}(\mathbf{0}, \Sigma)\right),
\end{aligned}
$$

where the constituents on the right hand side are independent, and the matrix $\Sigma$ is given by $\Sigma_{i, j}=\frac{1}{m+1}\left(\delta_{i, j}-\frac{1}{m+1}\right)$.

Let us compare these results to our findings above. We obtain from Lemma 2.14:

$$
\mathbf{E}\left(Y^{N}\right)=\frac{1}{4} \frac{m}{m+1} N^{2}-\frac{1}{4} \frac{m}{m+1} N,
$$

which agrees with the expectation given in Theorem 3.6. Further, we have

$$
\mathbf{E}\left(J_{i}^{N}\right)=i \frac{N}{m+1}, \quad b_{i}=\frac{i}{m+1}, \quad a_{0}=\ldots=a_{m-1}=0, a_{m}=1,
$$


and find that

$$
C^{N}=\frac{m(m+1)}{4} N^{2}
$$

and

$$
v(\mathbf{a}, \mathbf{b})=\frac{1}{12(m+1)^{3}} \sum_{i=1}^{m} i(i+1)=\frac{1}{36} \frac{m(m+2)}{(m+1)^{2}} .
$$

Finally, $\mathbf{c}=\mathbf{0}$, where $\mathbf{c}=\mathbf{c}(\mathbf{a}, \mathbf{b})$ is as in Corollary 2.11. Thus, by Corollary 2.11 we have

$$
\frac{Y^{N}-C^{N}}{N^{3 / 2}} \stackrel{d}{\longrightarrow} \mathcal{N}\left(0, \frac{1}{36} \frac{m(m+2)}{(m+1)^{2}}\right),
$$

which is equivalent to the weak convergence assertion in Theorem 3.6, and hence establishes an independent proof. Moreover, by Corollary 2.11

$$
\frac{e\left(\mathbf{L}^{N}, \mathbf{J}^{N}\right)-C^{N}}{N^{3 / 2}} \stackrel{d}{\longrightarrow} \mathcal{N}(0,0)=\delta_{0},
$$

that together with Theorem 2.1 independently proves Janson's Theorem 3.7.

Note that Theorem 3.7 as it stands does not generalize to more general distributions. As an example let $\mathbf{B}^{N}$ be multinomial with parameters $N$, $\mathbf{p}$ where $\mathbf{p}$ is not uniform. Here we get from Corollary 2.11 that

$$
\frac{e\left(\mathbf{L}^{N}, \mathbf{J}^{N}\right)-C^{N}}{N^{3 / 2}} \stackrel{d}{\longrightarrow} \mathcal{N}\left(0, v_{1}(\mathbf{p})\right)
$$

where $v_{1}(\mathbf{p})=\frac{1}{4}\left(\sum_{i=0}^{m} p_{i}^{3}-\left(\sum_{i=0}^{m} p_{i}^{2}\right)^{2}\right)$. The corresponding joint limiting distribution (on the right hand side of (37)) is normal, but the constituents are not independent.

\subsubsection{The unrestricted two component case}

For the two component case of $\mathbf{L}=\left(L_{1}, \ldots, L_{m}\right)$, i.e. $L_{m}=M, L_{k}=K$ for a $k<m$, and all other $L_{i}=0$, we find

$$
\begin{aligned}
\mathbf{E}\left(T_{\mathbf{L}}\right)= & \frac{1}{12} \frac{m(4 m+5)}{m+1} M^{2}+\frac{1}{12} \frac{m(2 m+1)}{m+1} M+\frac{1}{12} \frac{k(4 k+5)}{k+1} K^{2} \\
& +\frac{1}{12} \frac{k(2 k+1)}{k+1} K+\frac{1}{2} m K M+\frac{1}{6} \frac{k(k+2)}{m+1} K M .
\end{aligned}
$$

For $K=1$ this simplifies to

$$
\mathbf{E}\left(T_{\mathbf{L}}\right)=\frac{1}{12} \frac{m(4 m+5)}{m+1} M^{2}+\frac{1}{12} \frac{m(8 m+7)+2 k(k+2)}{m+1} M+\frac{k}{2} .
$$

Let us interpret this in terms of the Demazure modules $V_{w}\left(m \Lambda_{0}+n \Lambda_{1}\right)$ from (30). The random variables $X_{w}$ having probability generating function the basic specialization of 
the character $\chi\left(V_{w}(\Lambda)\right)$ are given due to Proposition 3.5 by translations and rotations (averaging over the random variable $S_{\mathbf{L}_{w}}$ ) as follows

Equations (32) and (33) read as:

$$
X_{w}=N^{2} m+N(N+1) n+(-2 N-1) \cdot S_{\mathbf{L}_{w}}+T_{\mathbf{L}_{w}} .
$$

Equations (34) and (35) read as:

$$
X_{w}=N^{2} m+N(N-1) n-2 N \cdot S_{\mathbf{L}_{w}}+T_{\mathbf{L}_{w}} .
$$

The cases covered here correspond to the cases found in [3, Theorem 4.1]. Let us restrict for simplicity reasons to (39) for $w=\left(s_{1} s_{0}\right)^{N}$, and compare our findings to [3, Theorem $4.1]$, where the corresponding case is $[3,(4.1)]$ for even $N$.

Theorem 3.8 ([3, (4.1) in Theorem 4.1]). For $\mathbf{L}$ with entries 0 except $L_{m}=1, L_{m+n}=$ $2 N-1$, and with $U=2 N-1, u=m+n$ one has

$$
\begin{aligned}
& \mathbf{E}\left(N^{2} m+N(N-1) n-2 S_{\mathbf{L}} N+T_{\mathbf{L}}\right) \\
& \quad=\frac{2 U m(m+2)+U(U-1) u(u+2)}{12(u+1)}+\frac{U-1}{2} \frac{u}{2}+\frac{m}{2} .
\end{aligned}
$$

Proof. We can establish (40) by the computation of the left-hand side through the linearity of $\mathbf{E}($.$) and the mean of the random variables S_{\mathbf{L}}$ and $T_{\mathbf{L}}$. That is

$$
\mathbf{E}\left(T_{\mathbf{L}}\right)=\frac{1}{12} \frac{u(4 u+5)}{u+1} U^{2}+\frac{1}{12} \frac{u(8 u+7)+2 m(m+2)}{u+1} U+\frac{m}{2},
$$

and $\mathbf{E}\left(S_{\mathbf{L}}\right)=\frac{1}{2}\left(m L_{m}+u L_{u}\right)=\frac{1}{2}(m+u U)=\frac{1}{2}(2 u N-n)$ which gives

$$
\mathbf{E}\left(N^{2} m+N(N-1) n-2 S_{\mathbf{L}} N\right)=-N^{2} u=-\frac{(U+1)^{2}}{4} u .
$$

Finally, we settle a question that was posed by Bliem and Kousidis in [5].

Lemma 3.9 (Cf. [5, Conjecture 8.3]). Fix a dominant integral weight $\Lambda$ and a sequence $\left(w_{N}\right)$ in $W^{\text {aff }}$ such that $l\left(w_{N}\right) \rightarrow \infty$. Let $\mu_{N}$ be the joint distribution of the degree and the finite weight in $V_{w_{N}}(\Lambda)$. Let $\tilde{\mu}_{N}$ be the distribution obtained from $\mu_{N}$ by normalizing to a probability distribution and rescaling the two coordinates individually so that $\operatorname{supp}\left(\tilde{\mu}_{N}\right)$ just fits into the rectangle $[0,1] \times[-1,1]$. Then, as $N \rightarrow \infty$,

$$
\tilde{\mu}_{N} \stackrel{\mathrm{w}}{\longrightarrow} \delta_{\left(\frac{\langle c, \Lambda\rangle+2}{3(\langle c, \Lambda\rangle+1)}, 0\right)},
$$

where $c=\alpha_{0}^{\vee}+\alpha_{1}^{\vee}$ denotes the canonical central element. 
Proof. We consider only the Demazure modules $V_{\left(s_{1} s_{0}\right)^{N}}\left(m \Lambda_{0}+n \Lambda_{1}\right)$ as the other cases can be derived similarly. Let $r_{N}$ denote the maximal degree in these Demazure modules, i.e. $r_{N}=N^{2} m+N(N-1) n$. Let $u=m+n=\langle c, \Lambda\rangle$ denote the level of the representation, and consider the random variable with probability generating function given by the basic specialization of our Demazure module, that is

$$
\begin{aligned}
X_{N} & =r_{N}-2 N S_{\mathbf{L}^{N}}+T^{N} \\
& =\mathbf{E}\left(r_{N}-2 N \mathbf{E}\left(S_{\mathbf{L}^{N}}\right)+T^{N}\right)-2 N\left(S_{\mathbf{L}^{N}}-\mathbf{E}\left(S_{\mathbf{L}^{N}}\right)\right)+\left(T^{N}-\mathbf{E}\left(T^{N}\right)\right)
\end{aligned}
$$

The probability distribution of $X_{N}$ and $\frac{1}{r_{N}} X_{N}$ is the first coordinate of $\mu_{N}$ and $\tilde{\mu}_{N}$ for the Weyl group element $w_{N}=\left(s_{1} s_{0}\right)^{N}$, respectively. Now, equivalent to the asserted weak convergence of $\tilde{\mu}_{N}$ we have

$$
\frac{X_{N}}{r_{N}} \stackrel{d}{\longrightarrow} \frac{u+2}{3(u+1)}
$$

since

$$
\frac{\mathbf{E}\left(r_{N}-2 N S_{\mathbf{L}^{N}}+T^{N}\right)}{r_{N}} \rightarrow \frac{1}{3} \frac{4 u+5}{u+1}-1=\frac{u+2}{3(u+1)},
$$

and by (11) and Corollary 2.11 we have the convergences in distribution

$$
\frac{S_{\mathbf{L}^{N}}-\mathbf{E}\left(S_{\mathbf{L}^{N}}\right)}{N} \stackrel{d}{\longrightarrow} 0, \quad \text { and } \quad \frac{T^{N}-\mathbf{E}\left(T^{N}\right)}{N^{2}} \stackrel{d}{\longrightarrow} 0 .
$$

Since it is well known that the second coordinate of $\tilde{\mu}_{N}$ concentrates in 0 , the claim follows.

\section{Fusion of symmetric power representations}

The Kostka numbers are the coefficients in the expansion

$$
\prod_{i} h_{\xi_{i}}(\mathbf{x})=\sum_{\xi} K_{\eta, \xi} \cdot s_{\eta}(\mathbf{x})
$$

of the product of complete symmetric functions $h_{\xi_{i}}$ in terms of the Schur functions $s_{\eta}$. The Kostka polynomials $K_{\eta, \mu}(q)$ generalize the Kostka numbers in the sense that $K_{\eta, \mu}(1)=$ $K_{\eta, \mu}$. They give the transition matrix between the Schur function $s_{\eta}$ and Hall-Littlewood function $P_{\mu}$, i.e.

$$
s_{\eta}(\mathbf{x})=\sum_{\mu} K_{\eta, \mu}(q) \cdot P_{\mu}(\mathbf{x}, q)
$$

A standard reference for the above functions is [23]. 
Now, the $q$-supernomial $S_{\xi, \mu}(q)[16,20,25,26,27]$ is defined as the combination of (41) and (42), i.e. as the transition between the above product of complete symmetric functions and Hall-Littlewood functions

$$
S_{\xi, \mu}(q)=\sum_{\eta} K_{\eta, \xi} \cdot K_{\eta, \mu}(q)
$$

An explicit form of $S_{\xi, \mu}(q)$ is proven in [16, Proposition 5.1], where $\mu=\left(\mu_{1}, \ldots, \mu_{m}\right)$ is a partition and $\xi \in \mathbf{Z}_{+}^{n}$ a composition such that $|\mu|=|\xi|=M$, as

$$
S_{\xi, \mu}(q)=\sum_{\{\nu\}} q^{\phi(\{\nu\})} \prod_{\substack{1 \leqslant a \leqslant n-1 \\
1 \leqslant i \leqslant \mu_{1}}}\left[\begin{array}{c}
\nu_{i}^{(a+1)}-\nu_{i+1}^{(a)} \\
\nu_{i}^{(a)}-\nu_{i+1}^{(a)}
\end{array}\right]_{q},
$$

with

$$
\phi(\{\nu\})=\sum_{a=0}^{n-1} \sum_{i=1}^{\mu_{1}}\left(\begin{array}{c}
\nu_{i}^{(a+1)}-\nu_{i}^{(a)} \\
2
\end{array}\right),
$$

and where the sum $\sum_{\{\nu\}}$ is indexed over the sequences of Young diagrams $\nu^{(1)}, \ldots, \nu^{(n-1)}$ such that

$$
\begin{aligned}
& \emptyset \subset \nu^{(0)} \subset \nu^{(1)} \subset \cdots \subset \nu^{(n-1)} \subset \nu^{(n)}=\mu^{t}, \\
& \left|\nu^{(a)}\right|=\xi_{1}+\cdots+\xi_{a} \text { for } 1 \leqslant a \leqslant n-1 .
\end{aligned}
$$

Remark 4.1. For $n=2$ and arbitrary $\mu$, (43) agrees with the definition of $q$-supernomials as given by Schilling and Warnaar [26]. See [20, §3.1] for a detailed discussion.

We define a slight variant of the above $q$-supernomials, which describes the string functions in the fusion product of $\mathfrak{s l}_{r+1}$ symmetric power representations $\mathcal{F}_{\mu}=V_{\mu_{1} \omega_{1}} *$ $V_{\mu_{2} \omega_{1}} * \ldots * V_{\mu_{m} \omega_{1}}$. That is,

$$
S_{\xi, \mu}^{*}(q)=q^{n(\mu)} S_{\xi, \mu}\left(q^{-1}\right)=q^{n(\mu)} \sum_{\eta} K_{\eta, \xi} \cdot K_{\eta, \mu}\left(q^{-1}\right),
$$

where for the partition $\mu=\left(\mu_{1}, \ldots, \mu_{m}\right)$ we set $n(\mu)$ (Cf. [19, (3.10)], [21, §2.1], [26, §2.1]) to be the normalization constant

$$
n(\mu)=\sum_{i=1}^{m}(i-1) \mu_{i}=\sum_{1 \leqslant i<j \leqslant m} \min \left(\mu_{i}, \mu_{j}\right) .
$$

Note that this normalization ensures that $q^{n(\mu)} K_{\eta, \mu}\left(q^{-1}\right)$ is a polynomial in $q$.

Then, we have a fermionic formula (a positive sum of products of $q$-binomial coefficients) for the graded character of the above fusion product $\mathcal{F}_{\mu}$. That is,

Proposition 4.2. Let $\mu=\left(\mu_{1}, \ldots, \mu_{m}\right)$ be a partition of $M$. Then,

$$
\chi\left(\mathcal{F}_{\mu}\right)=\sum_{\xi \text { weight }} S_{\xi, \mu}^{*}(q) \cdot m_{\xi}
$$


Proof. Let $m_{\xi}$ denote the monomial symmetric functions. Then, with the translation $\widetilde{K}_{\eta, \mu}(q)=q^{n(\mu)} K_{\eta, \mu}\left(q^{-1}\right)$ where $n(\mu)=\sum_{i}(i-1) \mu_{i}$ as in [19, (3.10)] one has [19, Corollary 7.6]:

$$
\begin{aligned}
\chi\left(\mathcal{F}_{\mu}\right) & =\sum_{\eta \vdash M} \chi\left(\pi_{\eta}\right) \cdot \widetilde{K}_{\eta, \mu}(q) \\
& =\sum_{\eta \vdash M} s_{\eta} \cdot \widetilde{K}_{\eta, \mu}(q) \\
& =\sum_{\eta \vdash M}\left(\sum_{\xi \text { weight }} K_{\eta, \xi} \cdot m_{\xi}\right) \cdot \widetilde{K}_{\eta, \mu}(q) \\
& =\sum_{\xi \text { weight }}\left(\sum_{\eta \vdash M} K_{\eta, \xi} \cdot \widetilde{K}_{\eta, \mu}(q)\right) \cdot m_{\xi} \\
& =\sum_{\xi \text { weight }} S_{\xi, \mu}^{*}(q) \cdot m_{\xi}
\end{aligned}
$$

Note that all partitions except $\mu$ have at most $r$ entries, corresponding to the rank of the Lie algebra.

Remark 4.3. For the graded character of fusion of fundamental representations $*_{j} V\left(\omega_{i_{j}}\right)$, Chari and Loktev prove an equivalent fermionic formula [8, Proposition 2.1.4].

Remark 4.4 (Cf. [4, 17, 22, 32]). Kirillov [20, 21] is a great source of various combinatorial, geometric and statistical interpretations of $q$-supernomials $S_{\xi, \mu}(q)$. Let us shortly remark on the geometric one. As pointed out by Kirillov [20, $\S 1.4]$ it has been proven by Shimomura [28] that the $q$-supernomials count the number of rational points $\mathrm{Fl}_{\xi}^{\mu}\left(\mathbf{F}_{q}\right)$ over the finite field $\mathbf{F}_{q}$ of the unipotent partial flag variety $\mathrm{Fl}_{\xi}^{\mu}$. To be precise, for a composition $\xi \in \mathbf{Z}_{+}^{r}$ of $n$, a $\xi$-flag in a $n$-dimensional vector space $V$ is a sequence $V_{1} \subset \cdots \subset V_{r}$ such that $\operatorname{dim} V_{i}=\xi_{1}+\cdots+\xi_{i}$. The set of all such flags is the partial flag variety $\mathrm{Fl}_{\xi}$. We let $\mathrm{Fl}_{\xi}^{\mu} \subset \mathrm{Fl}_{\xi}$ be the subset of the partial flag variety $\mathrm{Fl}_{\xi}$ consisting of the set of all $\xi$-flags $F \in \mathrm{Fl}_{\xi}$ fixed by a unipotent endomorphism $u \in \mathrm{Gl}(V)$ of type $\mu$ (a partition of $n$ that describes the Jordan canonical form of $u$ ). Then, $\mathrm{Fl}_{\xi}^{\mu}$ is a closed subvariety of $\mathrm{Fl}_{\xi}$, the so-called unipotent partial flag variety. Now, Shimomura [28] proves that the $q$-supernomials count the number of $\mathbf{F}_{q}$-rational points in $\mathrm{Fl}_{\xi}^{\mu}$. That is, with $n(\mu)$ as in (46) one has

$$
\# \mathrm{Fl}_{\xi}^{\mu}\left(\mathbf{F}_{q}\right)=q^{n(\mu)} S_{\xi, \mu}\left(q^{-1}\right)=S_{\xi, \mu}^{*}(q)
$$

In particular, the basic specialization of the fusion module $\mathcal{F}_{\mu}$ gives the number of $\mathbf{F}_{q^{-}}$ rational points in $\coprod_{\xi} \mathrm{Fl}_{\xi}^{\mu}$,

$$
\chi\left(\mathcal{F}_{\mu}\right)(q)=\sum_{\xi} S_{\xi, \mu}^{*}(q)=\sum_{\xi} \# \mathrm{Fl}_{\xi}^{\mu}\left(\mathbf{F}_{q}\right) .
$$


Our Proposition 4.2 exhibits the objects that have to be analyzed in order to establish a general central limit theorem along the same lines as Theorem 3.1. The explicit expression (45) shows that one can interpret the $q$-supernomials again as mixtures of probability distributions. For an Ansatz let

$$
\begin{aligned}
f_{\mu, \eta}(q) & =\frac{q^{n(\mu)} K_{\eta, \mu}\left(q^{-1}\right)}{K_{\eta, \mu}}, \\
\mathbf{P}\left(X_{\mu, \xi}=\eta\right) & =\frac{K_{\eta, \xi} K_{\eta, \mu}}{\sum_{\eta} K_{\eta, \xi} K_{\eta, \mu}} .
\end{aligned}
$$

Here, $f_{\mu, \eta}(q)$ would take the place of the inversion statistic, and $X_{\mu, \xi}$ of the mixing distribution. It should be straightforward to check the reductions to the distributions investigated in $\S 2$ in the case of $q$-supernomials as defined by Schilling and Warnaar (see Remark 4.1). We pose a conjecture for further research.

Conjecture 4.5. Consider the sequence of fusion modules of symmetric power representations for the current algebra $\mathfrak{s l}_{r} \otimes \mathbf{C}[t]$

$$
\mathcal{F}_{\mu^{N}}=V_{\omega_{1}}^{* L_{1}^{N}} * V_{2 \omega_{1}}^{* L_{2}^{N}} * \ldots * V_{m \omega_{1}}^{* L_{m}^{N}}
$$

associated to the partition $\mu^{N}=\left(1^{L_{1}^{N}}, 2^{L_{2}^{N}}, \ldots, m^{L_{m}^{N}}\right)$ with $L_{i}^{N}$-many $i$ 's. Assume that as $N \rightarrow \infty$ we have

$$
\frac{1}{N}\left(L_{1}^{N}, L_{2}^{N}, \ldots, L_{m}^{N}\right) \rightarrow \mathbf{a} \neq 0 .
$$

Then, the central string functions and the basic specialization of the character $\chi\left(\mathcal{F}_{\mu^{N}}\right)$ behaves asymptotically normal as $N \rightarrow \infty$.

\section{Acknowledgements}

The first author would like to thank Evgeny Feigin for helpful discussions.

\section{References}

[1] Patrick Billingsley. Probability and measure. Wiley, third edition, 1995.

[2] Patrick Billingsley. Convergence of probability measures. Wiley Series in Probability and Statistics: Probability and Statistics. John Wiley \& Sons Inc., New York, second edition, 1999. A Wiley-Interscience Publication.

[3] Thomas Bliem and Stavros Kousidis. Expected degree of weights in Demazure modules of $\widehat{\mathfrak{s l}}_{2}$. Transform. Groups, 16(4):1009-1025, 2011. doi:10.1007/s00031-011-9129-6.

[4] Thomas Bliem and Stavros Kousidis. The number of flags in finite vector spaces: Asymptotic normality and Mahonian statistics. J. Algebraic Combin., 37(2):361380, 2013. doi:10.1007/s10801-012-0373-1. 
[5] Thomas Bliem and Stavros Kousidis. On the law of large numbers for Demazure modules of $\widehat{\mathfrak{s l}}_{2}$. Asian-European J. Math., 6(2), 2013. doi : 10.1142/S1793557113500034.

[6] Rodney Canfield, Svante Janson, and Doron Zeilberger. The Mahonian probability distribution on words is asymptotically normal. Adv. in Appl. Math., 46:109-124, 2011.

[7] Roger Carter. Lie algebras of finite and affine type, volume 96 of Cambridge Studies in Advanced Mathematics. Cambridge University Press, Cambridge, 2005.

[8] Vyjayanthi Chari and Sergey Loktev. Weyl, Demazure and fusion modules for the current algebra of $\mathfrak{s l}_{r+1}$. Adv. Math., 207(2):928-960, 2006.

[9] Boris Feigin and Evgeny Feigin. $Q$-characters of the tensor products in $\mathfrak{s l}_{2}$-case. Mosc. Math. J., 2(3):567-588, 2002.

[10] Boris Feigin and Evgeny Feigin. Integrable $\widehat{\mathfrak{s l}}_{2}$-modules as infinite tensor products. In Fundamental mathematics today (Russian), pages 304-334. Nezavis. Mosk. Univ., Moscow, 2003.

[11] Boris Feigin and Sergey Loktev. On generalized Kostka polynomials and the quantum Verlinde rule. In Differential topology, infinite-dimensional Lie algebras, and applications, volume 194 of Amer. Math. Soc. Transl. Ser. 2, pages 61-79. Amer. Math. Soc., Providence, RI, 1999.

[12] William Feller. An introduction to probability theory and its applications. Vol. I. Third edition. John Wiley \& Sons Inc., New York, 1968.

[13] William Feller. An introduction to probability theory and its applications. Vol. II. Second edition. John Wiley \& Sons Inc., New York, 1971.

[14] Philippe Flajolet and Robert Sedgewick. Analytic combinatorics. Cambridge University Press, 2008.

[15] Ghislain Fourier and Peter Littelmann. Weyl modules, Demazure modules, KRmodules, crystals, fusion products and limit constructions. Adv. Math., 211(2):566593, 2007.

[16] Goro Hatayama, Anatol Kirillov, Atsuo Kuniba, Masato Okado, Taichiro Takagi, and Yasuhiko Yamada. Character formulae of $\widehat{\mathfrak{s l}}_{n}$-modules and inhomogeneous paths. Nuclear Phys. B, 536(3):575-616, 1999.

[17] Svante Janson. Generalized Galois numbers, inversions, lattice paths, Ferrers diagrams and limit theorems. The Electronic Journal of Combinatorics, 19(3):\#P34, 2012.

[18] Victor Kac. Infinite-dimensional Lie algebras. Cambridge University Press, third edition, 1990.

[19] Rinat Kedem. Fusion products, cohomology of $\mathrm{GL}_{N}$ flag manifolds, and Kostka polynomials. Int. Math. Res. Not., (25):1273-1298, 2004.

[20] Anatol Kirillov. New combinatorial formula for modified Hall-Littlewood polynomials. In q-series from a contemporary perspective (South Hadley, MA, 1998), volume 254 of Contemp. Math., pages 283-333. Amer. Math. Soc., Providence, RI, 2000. 
[21] Anatol Kirillov. Ubiquity of Kostka polynomials. Kirillov, Anatol (ed.) et al., Physics and combinatorics. Proceedings of the international workshop, Nagoya, Japan, August 23-27, 1999. Singapore: World Scientific. 85-200, 2001.

[22] Stavros Kousidis. Asymptotics of generalized Galois numbers via affine Kac-Moody algebras. Proc. Amer. Math. Soc., 141(10):3313-3326, 2013.

[23] Ian Macdonald. Symmetric functions and Hall polynomials. Oxford University Press, New York, 1995.

[24] Henry Mann and Donald Whitney. On a test of whether one of two random variables is stochastically larger than the other. Ann. Math. Statistics, 18:50-60, 1947.

[25] Anne Schilling. q-supernomial coefficients: from riggings to ribbons. In MathPhys odyssey, 2001, volume 23 of Prog. Math. Phys., pages 437-454. Birkhäuser Boston, Boston, MA, 2002.

[26] Anne Schilling and Ole Warnaar. Supernomial coefficients, polynomial identities and q-series. Ramanujan J., 2(4):459-494, 1998.

[27] Anne Schilling and Ole Warnaar. Inhomogeneous lattice paths, generalized Kostka polynomials and $A_{n-1}$ supernomials. Comm. Math. Phys., 202(2):359-401, 1999.

[28] Naohisa Shimomura. A theorem on the fixed point set of a unipotent transformation of the flag manifold. J. Math. Soc. Japan, 32:55-64, 1980.

[29] Anatolii Skorokhod. Limit theorems for stochastic processes. Teor. Veroyatnost. $i$ Primenen., 1:289-319, 1956.

[30] Lajos Takács. Some asymptotic formulas for lattice paths. J. Statist. Plann. Inference, 14:123-142, 1986.

[31] Tatsuya Tate and Steve Zelditch. Lattice path combinatorics and asymptotics of multiplicities of weights in tensor powers. J. Funct. Anal., 217(2):402-447, 2004.

[32] Ryan Vinroot. An enumeration of flags in finite vector spaces. Electron. J. Combin., 19(3):\#P5, 2012. 